\title{
Epidermal growth factor receptor inhibits colitis-associated cancer in mice
}

\author{
Philip E. Dubé, ${ }^{1,2}$ Fang Yan, ${ }^{2}$ Shivesh Punit, ${ }^{1}$ Nandini Girish, ${ }^{1}$ \\ Steven J. McElroy, ${ }^{2}$ M. Kay Washington, ${ }^{3}$ and D. Brent Polk ${ }^{1,2,4,5}$
}

\begin{abstract}
1The Saban Research Institute of Children's Hospital Los Angeles, Los Angeles, California, USA. 2Department of Pediatrics and ${ }^{3}$ Department of Pathology, Vanderbilt University School of Medicine, Nashville, Tennessee, USA. ${ }^{4}$ Department of Pediatrics and ${ }^{5}$ Department of Biochemistry and Molecular Biology, Keck School of Medicine, University of Southern California, Los Angeles, California, USA.
\end{abstract}

\begin{abstract}
Inflammatory bowel disease (IBD) is a chronic illness caused by complex interactions between genetic and environmental factors that propagate inflammation and damage to the gastrointestinal epithelium. This state of chronic inflammation increases the risk for development of colitis-associated cancer in IBD patients. Thus, the development of targeted therapeutics that can disrupt the cycle of inflammation and epithelial injury is highly attractive. However, such biological therapies, including those targeting epidermal growth factor receptor pathways, pose a risk of increasing cancer rates. Using two mouse models of colitis-associated cancer, we found that epidermal growth factor receptor inactivation accelerated the incidence and progression of colorectal tumors. By modulating inflammation and epithelial regeneration, epidermal growth factor receptor optimized the response to chronic inflammation and limited subsequent tumorigenesis. These findings provide important insights into the pathogenesis of colitis-associated cancer and suggest that epidermal growth factor-based therapies for IBD may reduce long-term cancer risk.
\end{abstract}

\section{Introduction}

Inflammatory bowel disease (IBD) is a major risk factor for colorectal cancer, so-called colitis-associated cancer (CAC), with increased tumor incidence and severity in ulcerative colitis and Crohn's disease (1-6). Thus, in developing therapies for IBD, not only the benefits for disease remission, but also the tumorigenic potential of such treatments must be considered. Indeed, this is a major concern for biological therapies, including those involving EGF or EGFR (ErbB1).

EGFR is a receptor tyrosine kinase that regulates multiple aspects of colon epithelial homeostasis, including proliferation, cell survival, wound closure, barrier function, and ion transport in order to optimize responses to injury (7-18). Within the colon, EGFR is expressed mainly on the basolateral aspect of all epithelial cells and, to a lesser extent, in subepithelial myofibroblasts and monocytic cells (19-21); however, the functional significance for EGFR in these latter two cell populations is unclear either in the normal or inflamed colon. Production of EGF, the prototypical EGFR ligand, is restricted to submandibular glands and Brunner's glands in the normal gastrointestinal tract $(22,23)$; however, in response to injury, the formation of ulcer-associated epithelial cell lineages may provide a localized source for EGF production in all regions of the gastrointestinal tract (24). The luminal release by these glands suggests that exocrine EGF only activates basolateral EGFR following epithelial injury and may therefore represent a so-called luminal surveillance factor (19). Unlike exocrine EGF, other EGFR ligands (TGF- $\alpha$, heparin-binding EGF, amphiregulin) are more widely distributed throughout the colon and are expressed by epithelial cells and myofibroblasts, suggesting that these ligands act in an autocrine or paracrine manner. This mode of signaling is of particular importance for EGFR transactivation by stimuli such

Conflict of interest: The authors have declared that no conflict of interest exists. Citation for this article: J Clin Invest. 2012;122(8):2780-2792. doi:10.1172/JCI62888 as TNF, bacterial products, and TLR4 activation, which activate EGFR either by ligand release or intracellular signaling pathways $(15,18,25)$; thus, these transactivation mechanisms constitute an important aspect of EGFR-mediated cytoprotection during inflammation. This emphasizes an underappreciated role for EGFR in regulating inflammatory outcomes, in particular during colitis, in which epithelial homeostasis and inflammation are dysregulated. Indeed, EGFR is protective in murine colitis models $(12,17,18)$, and, moreover, there is reduced EGFR signaling in IBD patients $(26,27)$, suggesting that impaired EGFR may contribute to disease etiology.

Thus, EGFR-directed therapies represent an attractive approach for IBD, and a single clinical trial by Sinha et al. provided promising results demonstrating the ability of EGF to induce and maintain remission in ulcerative colitis (28). However, a major criticism of this approach is that EGFR activation might accelerate tumorigenesis (29). Indeed, EGFR is widely considered a tumor promoter; its expression or activation is increased in many colonic precancerous lesions and tumors (30-32), and EGFR is implicated in animal models of gastrointestinal tumorigenesis (25, 33-36). Moreover, EGFR monoclonal antibodies are approved for metastatic colorectal cancer treatment (cetuximab and panitumumab) (37). However, EGFR inhibition is not universally efficacious, and resistance to EGFR inhibition occurs in tumors with mutant BRAF or KRAS (37-40). Importantly, these mutations are common in tumors from ulcerative colitis patients $(41,42)$, suggesting possible widespread resistance to EGFR inhibition in this population.

It is unclear how EGFR signaling might contribute to the etiology or prognosis of tumorigenesis in CAC (Supplemental Figure 1; supplemental material available online with this article; doi:10.1172/JCI62888DS1). Clinical data suggest that disease severity and duration correlate with cancer risk in IBD; thus, treatment goals should focus on inducing mucosal healing and minimizing inflammation to prevent CAC (1-6). On one hand, 
A
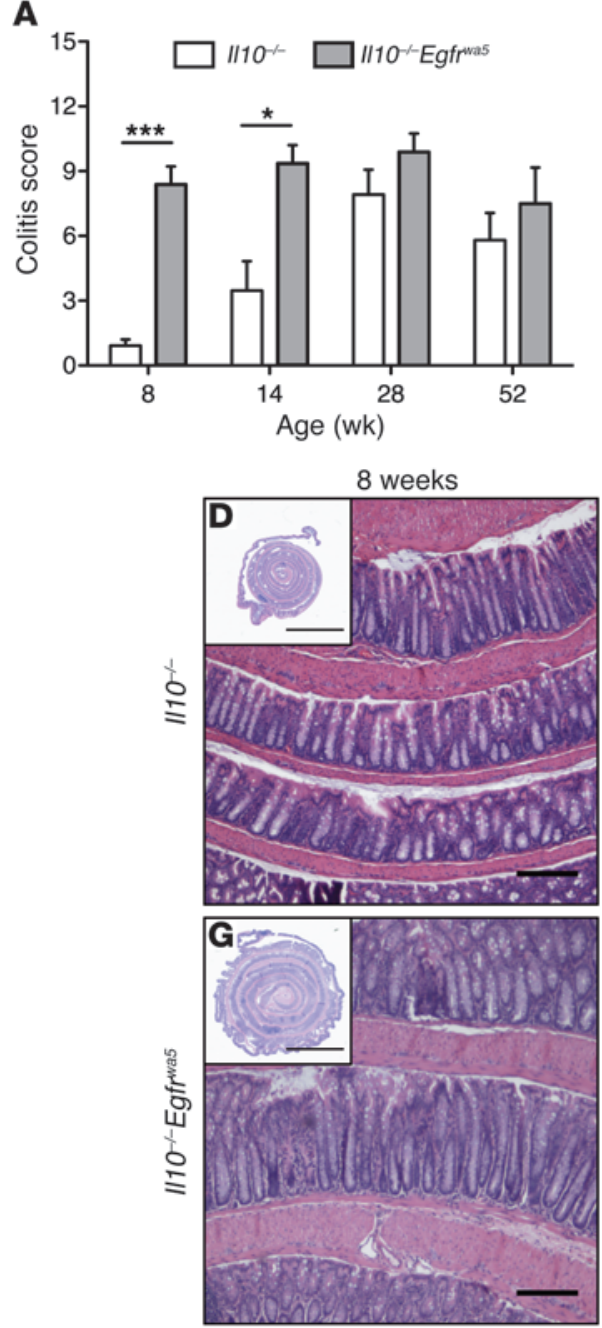

B
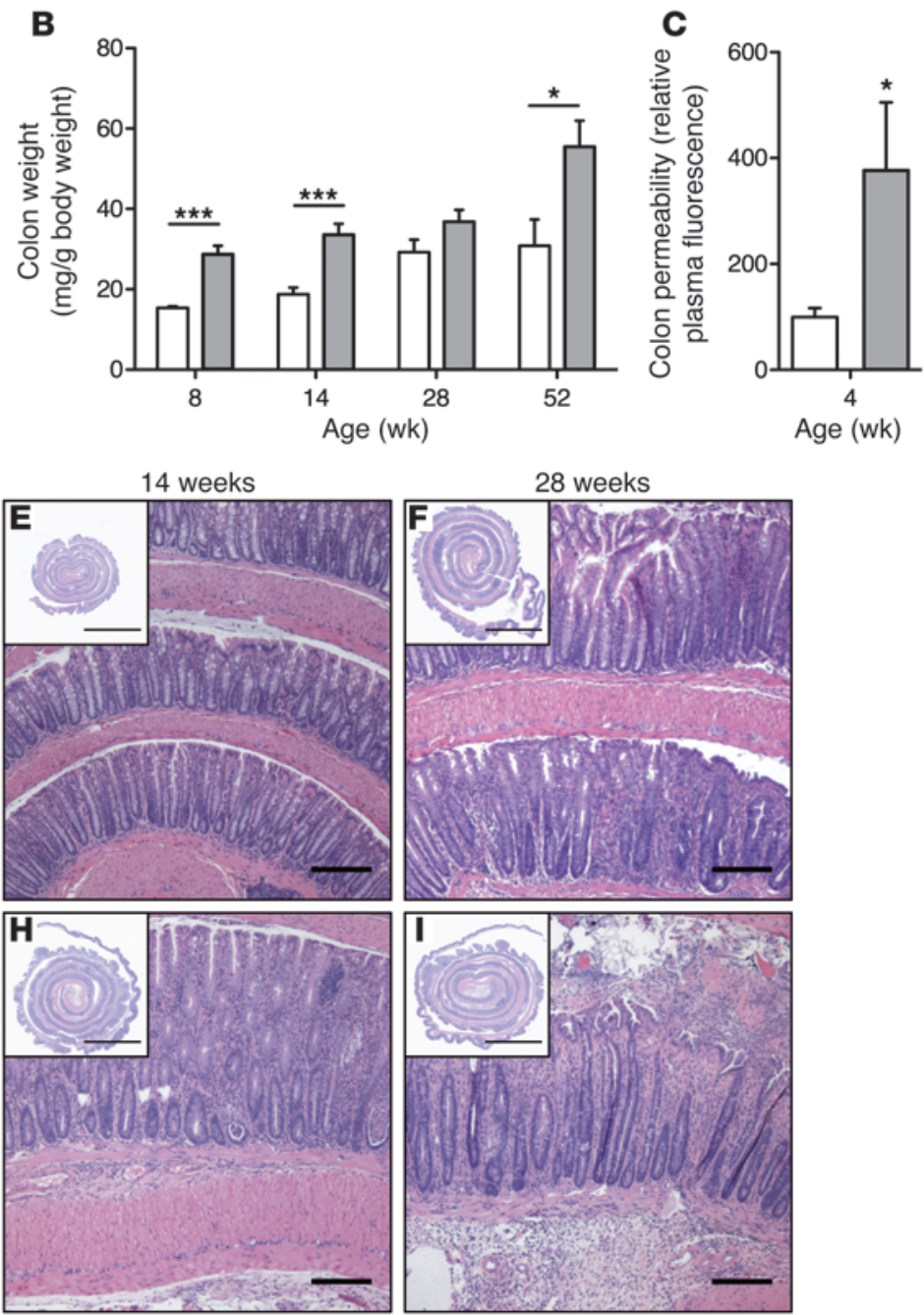

28 weeks
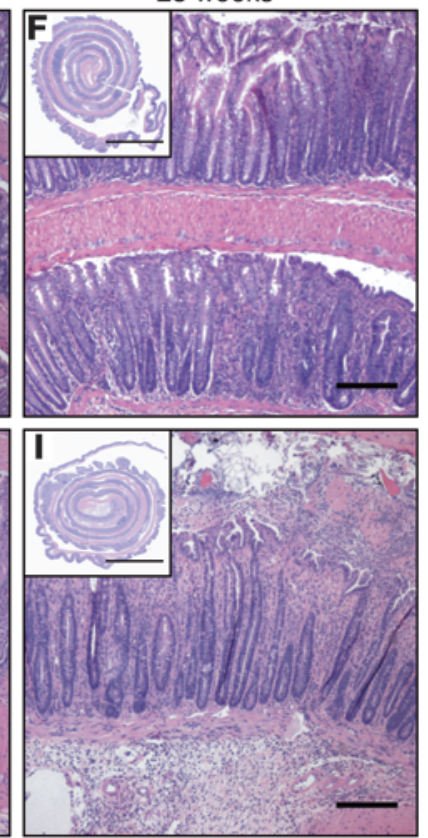

\section{Figure 1}

EGFR mitigates colitis in $/ / 10^{-/-}$mice. Colitis scores (A) and colon weights (B) of $/ / 10^{-/-}$and $/ / 10^{-/-E g f r w a 5}$ mice at the indicated ages; $n=6-15$; ${ }^{\star} P<0.05,{ }^{* * *} P<0.001$. (C) In vivo colon permeability assay of $I / 10^{-/-}$and $I / 10^{-/-}$Egfrwas littermates at 4 weeks of age as determined by FITC-dextran enema; $n=3$, ${ }^{\star} P<0.05$. (D-I) Representative H\&E colon sections from mice of the indicated genotypes and ages. Scale bars: $5 \mathrm{~mm}$, overview insets; $100 \mu \mathrm{m}$, high-power fields.

EGFR signaling may limit disease severity by minimizing injury and optimizing epithelial regeneration, and it may even regulate inflammatory outcomes. Indeed, according to data from animal studies and the short-term use of EGF enemas in ulcerative colitis patients, EGFR-targeted therapies show such potential $(12,17$, $18,28)$. However, given its association with tumorigenesis, EGFR activation may also potentiate the development or growth of subsequent tumors. Thus, the important remaining question is how EGFR-targeted therapies for IBD influence long-term cancer risk.

Here, we tested the hypothesis that EGFR inhibition prevents colon tumor development during colitis in two mouse models of CAC (43-46). Paradoxically, we show that EGFR inactivation in these models not only exacerbated colitis, but also dramatically and unexpectedly increased colon tumorigenesis. These results demonstrate an EGFR-independent pathway for tumorigenesis in chronic colitis and uncover a paradoxical tumor-suppressing role for EGFR through mitigation of colitis severity, inflammation, and subsequent tumorigenesis.

\section{Results}

EGFR inhibits colitis in $\mathrm{Il10}^{-/-}$mice. EGFR regulates multiple epithelial responses to injury; however, the role of EGFR in immunemediated colitis has not been examined. To address this, we used the clinically relevant $I l 10^{-/-}$model of spontaneous colitis (43-45, 47), which resembles many aspects of human IBD (45) and is particularly relevant to the etiology of IBD in patients with IL-10 signaling defects (48-51). Il10-/- mice were crossed to mice with a dominant-negative EGFR point mutation (Egfrwas mice, which show $\sim 95 \%$ EGFR inhibition) (52) to test the hypothesis that EGFR inhibits colitis (Figure 1). Il10-/-Egfrwas mice presented with severe colitis by 8 weeks of age, which was apparent by histology and by increased colitis scores and colon weights (indicative of colitis-induced hyperplasia). By 12 weeks, colitis was evident by colonoscopy (Supplemental Figure 2). In $I l 10^{-/-}$Egfrwa5 mice, colon weights continued to increase through 1 year of age, indicative of worsening colitis-induced hyperplasia. In contrast, compared with wild-type mice, $\mathrm{Il10}^{-/-}$mice did not show any histologic or colono- 


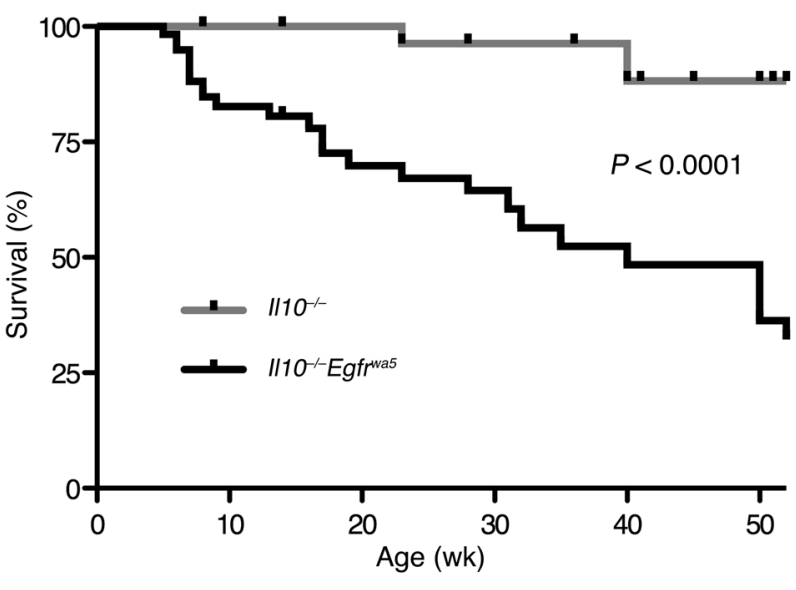

Figure 2

EGFR increases survival in $/ / 10^{-/-}$mice. Kaplan-Meier survival curves for $/ 110^{-/-}$and $/ / 10^{-/-E g f r w a 5}$ mice were compared by the log-rank test.

scopic evidence of colitis prior to 28 weeks. Colitis in Il10-/-Egfrwas mice was characterized by extreme epithelial hyperplasia and dysplasia, with increased inflammatory infiltrates and crypt abscesses. Indeed, this colitis was so severe that $I l 10^{-/}$Egfrwas mice had significantly lower survival compared with $I l 10^{-/-}$littermates (Figure 2). Upon necropsy, moribund $I l 10^{-/}$Egfrwa5 mice presented with distended colons, occlusions, and loose fecal contents, suggestive of advanced severe colitis. Thus, given higher mortality and early removal from this study, the data for $I l 10^{-/-E g f r w a s}$ mice at advanced ages (i.e., 28 and 52 weeks of age) likely underestimate the full penetrance of the colitis phenotype, given that those mice most severely affected succumbed before this age and are not included in the data presented herein. To determine the inflammatory characteristics underlying this phenotype, we examined the profile of inflammatory cells and cytokines in these colons. Although $\mathrm{Il10}^{-/-}$Egfrwas colons had increased inflammatory cell infiltration, the types of inflammatory cells present, expressed as a percentage of all cells, were not different from those in $\mathrm{Il10}^{-/-}$ colons (Supplemental Figure 3). Furthermore, the profile of cytokines released by colon explants was not different between Il10 $0^{-/}$and Il10-/- Egfrwa5 specimens, with each showing a combination of Th1 and Th17, but not Th2, cytokines (Supplemental Figure 4). We compared the expression of inflammation-related mRNA transcripts from 14-week-old colons (Table 1) and found that EGFR inactivation in the $1110^{-/-}$background increased Tnf, Il6, Il17a, and $I l 21$ expression, indeed indicative of increased Th1 and pathogenic Th17 responses. However, by 28 weeks of age, these differences abated (Table 2 ), potentially due to decreased $I l 10^{-/-}$Egfrwas survival or disease progression in $I l 10^{-/-}$controls. Thus, EGFR inactivation did not qualitatively alter the type of inflammation in $\mathrm{Il10}^{-/-}$colitis, but it certainly accelerated disease progression, with an early exacerbation of cytokine production. Interestingly, there was no evidence of inflammation or colitis in Egfrwas mice (Table 1 and Supplemental Figure 5), even at 1 year of age (data not shown). However, since EGFR inactivation increased colon permeability on both the $\mathrm{Il10^{+/+ }}$ and $I l 10^{-/-}$backgrounds (Figure $1 \mathrm{C}$ and Supplemental Figure 5, E and $F$ ), this suggests that an inherent permeability defect accelerated colitis in Il10-/-Egfrwas mice, consistent with the hypothesis that increased access to luminal antigens drives inflammation in this model (53). Given that we found no evidence of abnormal tight junction structure in the Egfrwas colon epithelium by electron microscopy or distribution of the tight junction proteins $\mathrm{ZO}-1$ and occludin (data not shown), this Egfrwas permeability defect likely relates to impaired physiological wound healing versus a tight junction defect per se. Increased colon $I l 10$ mRNA expression and a trend toward increased Foxp 3 expression in Egfrwas compared with wild-type mice (Table 1) suggest that protective tolerogenic mechanisms exist to prevent inflammation in spite of increased permeability; indeed, a similar mechanism has been postulated following targeted tight junction disruption (54). In contrast, the lack of IL-10-regulated immunoregulation in combination with increased permeability in $I l 10^{-/-}$Egfrwas mice likely accelerated Il10-/-associated colitis, without altering the type of subsequent inflammation. To confirm an inhibitory role of EGFR in $\mathrm{IllO}^{-/-}$ colitis, we employed an additional EGFR loss-of-function recessive mutation (Egfrwa2/wa2, $280 \%$ EGFR inhibition) (55). As with the $E G^{2} R^{\text {wa } 5}$ crosses, $I l 10^{-/-}$Egfrwa2/wa2 mice displayed a similar acceleration of colitis versus controls (Supplemental Figure 6).

We next examined epithelial responses upon EGFR inactivation, under normal conditions and during $I l 10^{-/-}$-induced colitis (Figure 3). Despite a well-recognized role for EGFR in inducing proliferation, the loss of EGFR signaling in $I l 10^{-/}$Egfrwas mice actually increased epithelial proliferation, as shown by the active cell cycle marker Ki-67 and the metaphase marker phosphorylated histone $\mathrm{H} 3$ ( $\mathrm{pH}-\mathrm{H} 3$ ). Indeed, this hyperproliferation was so dramatic that in several cases, proliferating surface epithelial cells could be readily identified, whereas epithelial proliferation in control $I l 10^{-/-}$mice was always confined to the crypt base. We confirmed that these $\mathrm{pH}-\mathrm{H}^{+}$cells were in metaphase by dual immunofluorescence staining for tubulin, a component of the
Table 1

Cytokine gene expression profile for 14-week-old colon tissue

\begin{tabular}{|c|c|c|c|c|c|c|}
\hline & Egfrt/+ & Egfrwa5 & $P$ & $1110^{-/-}$ & II10-/-Egfrwa5 & $P$ \\
\hline Ifng & $0.013 \pm 0.004$ & $0.013 \pm 0.004$ & 0.98 & $1.0 \pm 0.8$ & $0.8 \pm 0.2$ & 0.78 \\
\hline $1 / 6$ & $0.033 \pm 0.008$ & $0.022 \pm 0.004$ & 0.30 & $1.0 \pm 0.4$ & $6.9 \pm 2.7$ & 0.049 \\
\hline$/ / 10$ & $1.0 \pm 0.3$ & $2.0 \pm 0.3$ & 0.048 & n.d. & n.d. & \\
\hline $1 / 17 a$ & $0.002 \pm 0.001$ & $0.002 \pm 0.001$ & 0.66 & $1.0 \pm 0.6$ & $10.1 \pm 3.4$ & 0.018 \\
\hline II21 & $0.26 \pm 0.24$ & $0.20 \pm 0.07$ & 0.82 & $1.0 \pm 0.4$ & $7.2 \pm 1.1$ & 0.0001 \\
\hline $1123 a$ & $0.022 \pm 0.004$ & $0.017 \pm 0.006$ & 0.46 & $1.0 \pm 0.3$ & $0.6 \pm 0.1$ & 0.26 \\
\hline $\operatorname{Tgfb} 1$ & $0.014 \pm 0.001$ & $0.016 \pm 0.004$ & 0.76 & $1.0 \pm 0.2$ & $0.9 \pm 0.2$ & 0.60 \\
\hline Tnf & $0.3 \pm 0.1$ & $0.3 \pm 0.1$ & 0.96 & $1.0 \pm 0.4$ & $6.4 \pm 2.0$ & 0.013 \\
\hline Foxp3 & $0.03 \pm 0.03$ & $0.11 \pm 0.03$ & 0.11 & $1.0 \pm 0.4$ & $2.3 \pm 0.6$ & 0.066 \\
\hline Gata3 & $0.4 \pm 0.1$ & $0.5 \pm 0.1$ & 0.29 & $1.0 \pm 0.3$ & $3.1 \pm 0.6$ & 0.009 \\
\hline Tbet & $0.05 \pm 0.01$ & $0.12 \pm 0.06$ & 0.29 & $1.0 \pm 0.5$ & $1.0 \pm 0.4$ & 0.96 \\
\hline Egfr & $2.1 \pm 0.2$ & $1.4 \pm 0.4$ & 0.13 & $1.0 \pm 0.1$ & $3.3 \pm 0.8$ & 0.010 \\
\hline
\end{tabular}

Tissue mRNA transcripts were quantified by real-time RT-PCR and normalized to Actb. Data are presented relative to $/ / 10^{-/-}$values, except $/ / 10$ transcripts, which are presented relative to wild-type values; n.d. not determined, $n=4-9$. Significant values are indicated in boldface. 
Table 2

Cytokine gene expression profile for 28-week-old colon tissue

\begin{tabular}{lccc} 
& $\boldsymbol{I 1 \mathbf { 1 } ^ { - / }}$ & $\boldsymbol{I I 0}^{-/}$-Egfrwa5 & $\boldsymbol{P}$ \\
Ifng & $1.0 \pm 0.2$ & $1.6 \pm 0.4$ & 0.18 \\
II6 & $1.0 \pm 0.2$ & $5.9 \pm 2.9$ & 0.12 \\
II17a & $1.0 \pm 0.4$ & $0.5 \pm 0.1$ & 0.28 \\
II21 & $1.0 \pm 0.3$ & $1.5 \pm 0.4$ & 0.42 \\
II23a & $1.0 \pm 0.3$ & $0.9 \pm 0.1$ & 0.68 \\
Tgfb1 & $1.0 \pm 0.1$ & $0.9 \pm 0.1$ & 0.66 \\
Tnf & $1.0 \pm 0.2$ & $0.9 \pm 0.2$ & 0.76 \\
Foxp3 & $1.0 \pm 0.1$ & $1.0 \pm 0.1$ & 0.92 \\
Gata3 & $1.0 \pm 0.3$ & $1.5 \pm 0.2$ & 0.20 \\
Tbet & $1.0 \pm 0.3$ & $0.7 \pm 0.1$ & 0.26 \\
Egfr & $1.0 \pm 0.2$ & $0.9 \pm 0.02$ & 0.64 \\
\hline
\end{tabular}

Tissue mRNA transcripts were quantified by real-time RT-PCR and normalized to Actb. Data are presented relative to $/ 110^{-1-}$ values; $n=6$.

mitotic spindle apparatus, in which $\mathrm{pH}-\mathrm{H}^{+}$cells undergoing cytokinesis were readily apparent (Supplemental Figure 7A). Similar results were confirmed with in vivo BrdU labeling (representative figure in Supplemental Figure 7B). In contrast, EGFR inactivation in the absence of inflammation (Egfrwas vs. wild-type mice) reduced epithelial proliferation, although this effect was rather modest. Thus, rather than limiting epithelial proliferation in Il10-/- colitis, EGFR inactivation exacerbated this feature. Therefore, EGFR-induced proliferation is not likely the primary driver of the hyperproliferative response in $\mathrm{IllO}^{-/-}$colitis; alternatively, multiple cytokines might induce this response (e.g., TNF; ref. 56). In contrast, EGFR inactivation increased epithelial apoptosis, both with and without inflammation; nonetheless, this effect was greater during colitis (i.e., $450 \% \pm 50 \%$ increase in the $1 l 10^{-/-}$ background vs. $280 \% \pm 20 \%$ increase in the $I l 10^{+/+}$background). Interestingly, EGFR inactivation also increased the number of epithelial cells with signs of DNA damage (phospho-histone $\mathrm{H} 2 \mathrm{AX}$-positive) (57), both under normal conditions and during Ill10 ${ }^{--}$-induced inflammation, suggesting that EGFR signaling may reduce epithelial DNA damage or limit the production of genotoxic mediators in the colon. Thus, EGFR increases epithelial cell survival and limits DNA damage during inflammation, while preventing epithelial hyperproliferation; taken together, these effects on epithelial biology are likely mechanisms involved in the protective effects of EGFR in chronic colitis. Indeed, EGFR inactivation in $I l 10^{-/}$Egfrwas mice induced dramatic epithelial alterations, resulting in extensive dysplasia, suggesting that loss of EGFR might actually promote tumorigenesis in this model.

EGFR inhibits Il10-/- tumorigenesis. EGFR may play differential roles in CAC by either inducing colitis remission or promoting the development or progression of subsequent colorectal adenocarcinomas. Thus, we determined how EGFR inactivation influenced tumor development in the $I l 10^{-/-}$model. Although EGFR inactivation worsened colitis, we were surprised that it also exacerbated the incidence and progression of colon tumors (Figure 4). In this model, the resulting adenomas were elevated plaque-like lesions arising in a background of mucosal hyperplasia and inflammation. They were distinguished from hyperplasia by greater crowding and architectural complexity of crypts, with nuclear features of low-grade dysplasia, similar to the mucosal changes overlying invasive tumors. Further tumor progression was also evidenced by the presence of adenocarcinomas, identified by invasion of the submucosa or deeper tissues. By 14 weeks of age, loss of EGFR signaling increased both tumor incidence and progression, with $18 \%$ of $I l 10^{-1-}$ Egfrwa5 mice possessing adenocarcinomas invasive into the submucosa. This effect worsened with age, leading to increased tumor incidence and depth of invasion; indeed, by 1 year of age, $\mathrm{IllO}^{-/-} \mathrm{Eg} f \mathrm{rr}^{\mathrm{waS}}$ mice had a greater number of gross colon tumors (Figure 4B; no tumors were obvious at a macroscopic level at earlier time points examined), and one Il10-- Egfrwas mouse even presented with an adenocarcinoma invasive through the serosa into the perirectal fat (Figure $4 \mathrm{I}$ ). In contrast, Il10 $0^{--}$littermates did not demonstrate any microscopic evidence of invasive tumors at time points prior to 1 year of age (the incidence [\% of mice with invasive adenocarcinoma] in $I l 10^{-/-}$versus $I l 10^{-/-}$Egfrwas mice was: at 8 weeks $0 ; 14$ weeks: 0 versus $18 ; 28$ weeks: 0 versus 67; 52 weeks: 70 versus 100). Thus, EGFR inactivation in $\mathrm{IllO}^{-/-}$ mice increased the incidence and accelerated the progression of colorectal tumors. After formation, tumors in both $\mathrm{IlNO}^{-/-}$and $I l 10^{-/-E g f r w a s}$ mice appeared similar, both presenting as flat, non -polypoid adenomas or adenocarcinomas; however, these tumors nonetheless occurred earlier and were more numerous and aggressive in $I l 10^{-/-}$Egfrwas mice. To determine whether these tumors were particular to $I l 10^{-/-}$Egfrwas crosses, we also examined tumor formation in Il10-/-Egfrwa2/wa2 crosses (Supplemental Figure 6). These mice had increased tumor progression scores at 14 weeks of age, albeit to a lesser extent than those observed with Egfrwas crosses, potentially due to a lower level of EGFR inhibition with this mutation. Nevertheless, these data uncover a surprising tumor-suppressing role for EGFR in $I l 10^{-/-}$colitis.

EGFR inbibits AOM/DSS tumorigenesis. The Il10-/- CAC model is informative, given the presence of IL-10 signaling defects in IBD patients (48-51) and the similarities of this model to IBD with spontaneous tumor formation $(44,45)$. Nevertheless, to rule out idiosyncrasies of $I l 10^{-/-}$mice and to determine whether these findings were more widely applicable to CAC, we also tested whether EGFR inactivation accelerated tumorigenesis in an azoxymethane/ DSS (AOM/DSS) model (46), in which wild-type or Egfrwas mice received a single injection of the carcinogen AOM, followed by one round of DSS-induced colitis. Interestingly, EGFR inactivation did not affect the incidence of AOM/DSS-induced tumor formation (Figure 5A), but it significantly increased tumor progression (Figure $5 \mathrm{~B}$ ). Indeed, $40 \%$ of AOM/DSS-treated Egfrwas mice had invasive adenocarcinomas, while tumor invasion was not observed in wild-type mice, in which the tumors maintained a non-invasive polypoid structure. Moreover, colitis was worsened by EGFR inactivation, with a $44 \%$ mortality rate among Egfrwa5 mice during DSS administration, whereas no wild-type mice died during this period. These data differ from those of Dougherty et al., who showed that EGFR inactivation (Egfrwa2/wa2) reduced tumorigenesis following AOM/DSS, albeit only in the setting of diet-induced obesity (34). However, their conclusions are complicated by their use of mice on a mixed $\mathrm{A} / \mathrm{J} \times \mathrm{C} 57 \mathrm{BL} / 6$ background, since $\mathrm{A} / \mathrm{J}$ mice are exquisitely susceptible to AOM-induced colon tumorigenesis, even without an inflammatory stimulus (58). Nevertheless, this is intriguing given the associations between obesity and colorectal cancer (59), suggesting that lifestyle and genetic factors, in addition to inflammation, may alter the role of EGFR in colorectal tumorigenesis. While EGFR inactivation did not affect tumor incidence in our AOM/DSS study (Figure 5A), this likely occurred due to similar AOM-induced tumor initiation; since DSS-induced inflammation 
A

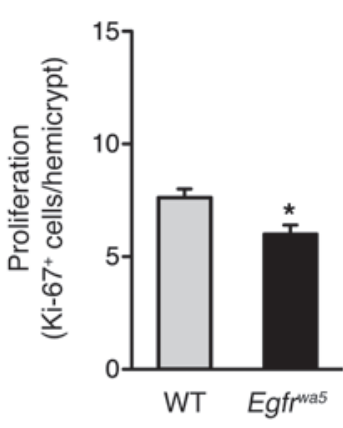

\section{B}
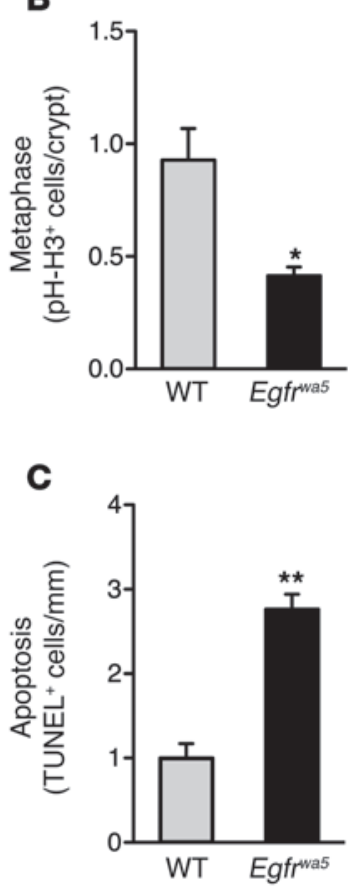

D

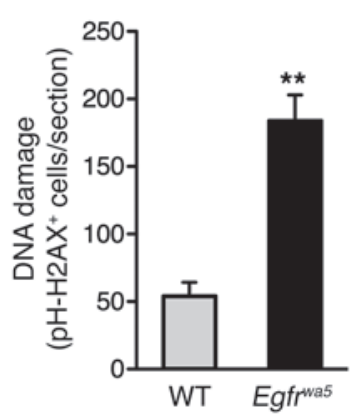

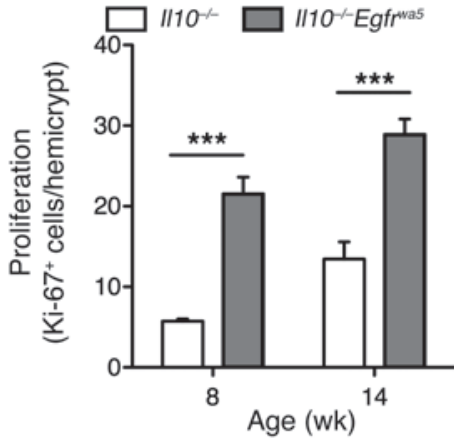
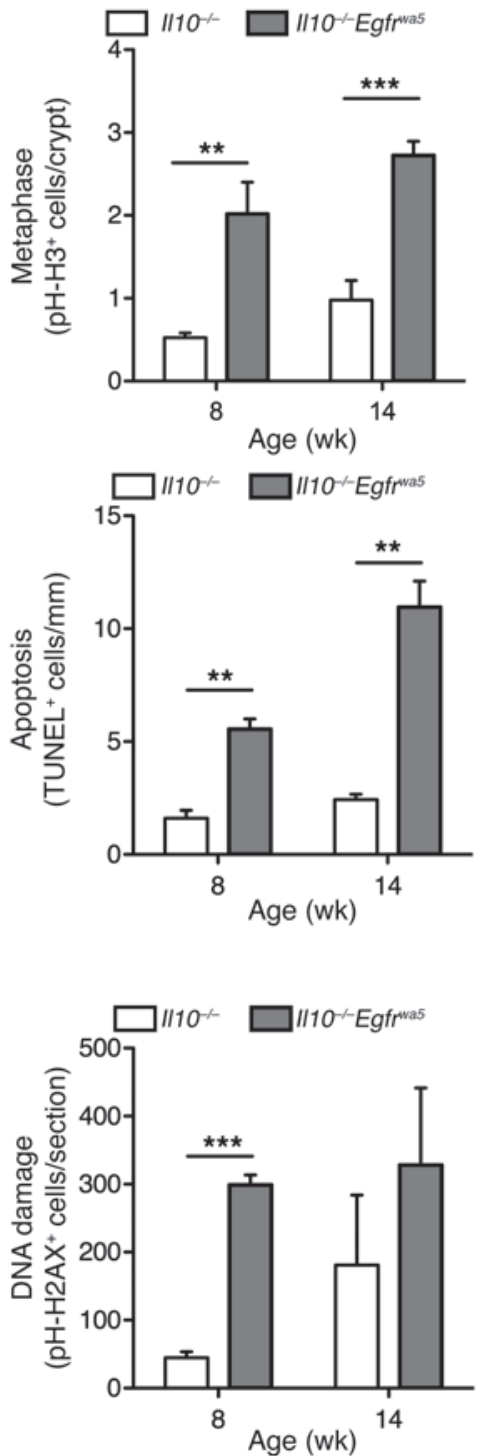

Ki-67

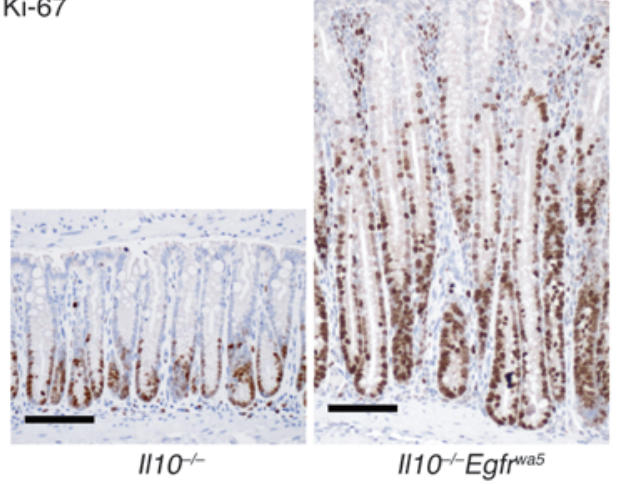

pH-H3

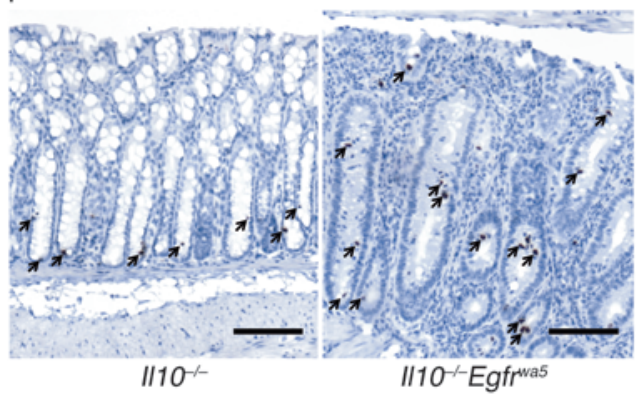

TUNEL

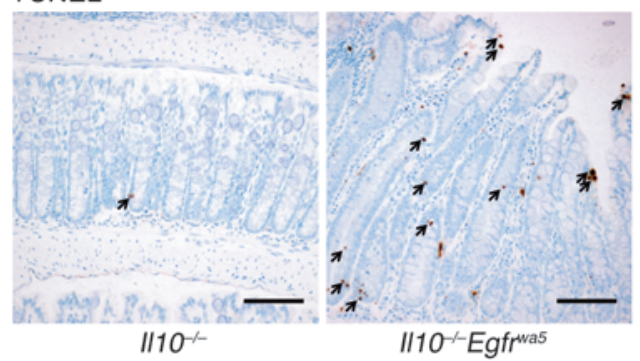

pH-H2AX

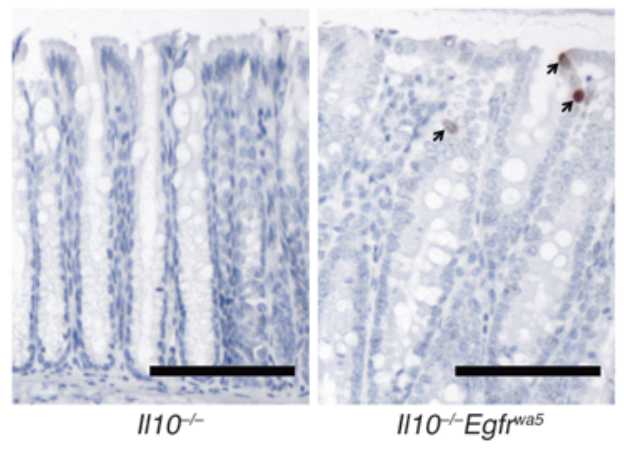

Figure 3

EGFR inhibition exacerbates epithelial cell proliferation, apoptosis, and DNA damage in $/ 110^{-/-}$colitis. (A and B) Quantification of epithelial (A) proliferation (Ki-67) and (B) metaphase indices (pH-H3); $n=9-15 ;{ }^{*} P<0.05,{ }^{* \star} P<0.01,{ }^{* * \star} P<0.001$. Representative images of 8-week-old mice are shown; scale bars: $50 \mu \mathrm{m}$. Cells positive for $\mathrm{pH}-\mathrm{H} 3$ are indicated with arrowheads. (C) Quantification of apoptosis $(\mathrm{TUNEL}) ; n=3 ;{ }^{* *} P<0.001$. Representative images of 14-week-old mice are shown; scale bars: $100 \mu \mathrm{m}$; arrows indicate TUNEL-positive epithelial cells. (D) Quantification of DNA damage (phospho-histone H2AX [pH-H2AX]); $n=3-6$; ${ }^{\star \star} P<0.01$, ${ }^{\star \star \star} P<0.001$. Representative images of 8-week-old mice are shown; scale bars: $100 \mu \mathrm{m}$; arrows indicate positive epithelial cells. 
A

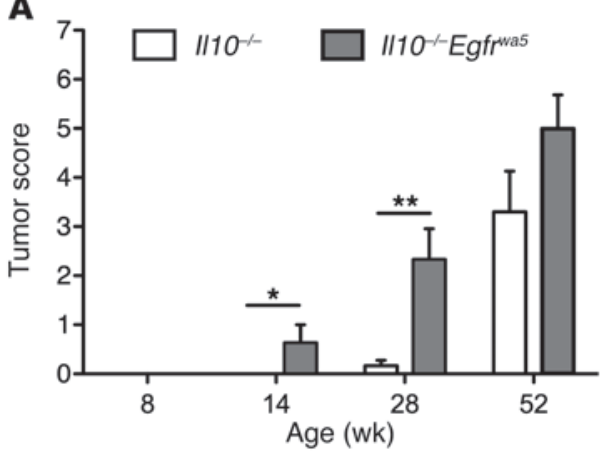

B

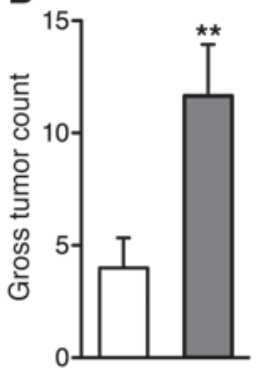

Figure 4

EGFR is a colon tumor suppressor in $/ 110^{-/-}$mice. (A) Tumor scores quantifying the microscopic incidence and progression of adenomas and adenocarcinomas from $/ / 10^{-/-}$and $/ / 10^{-/-}$Egfrwa5 mice at the indicated ages; $n=6-15 ;{ }^{*} P<0.05$, ${ }^{* *} P<0.01$. Scores indicate normal, 0 ; adenoma(s), 1-2; adenocarcinoma(s) with submucosal invasion, 3-4; adenocarcinoma(s) with muscularis invasion, 5-6; and adenocarcinoma(s) with serosal invasion, 7-8, as in Supplemental Table 2. (B) Macroscopic gross tumor counts from 1-year-old $/ / 10^{-/-}$and $/ / 10^{-/-}$Egfrwa5 colons; $n=6-10 ;{ }^{* *} P<0.01$. (C and D) Representative $\mathrm{H} \& \mathrm{E}$ colon section from a single 1-year-old $/ / 10^{-/-}$and $/ / 10^{-/-}$Egfrwa5 mouse; scale bars: $5 \mathrm{~mm}$. (E-H) Representative H\&E sections from different 1-year-old $/ 110^{-/-E g f r w a 5}$ colons showing dysplasia (E) and adenocarcinomas invasive into the submucosa $(\mathbf{F})$, muscularis propria $(\mathbf{G})$, and perirectal fat $(\mathbf{H})$; scale bars: $200 \mu \mathrm{m}$.
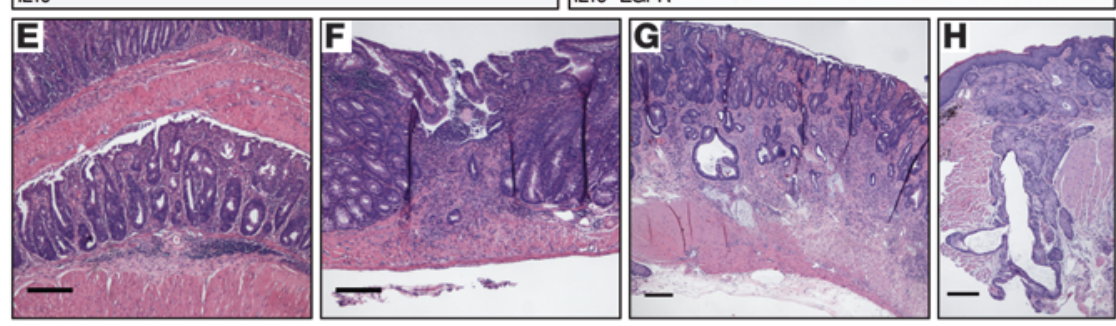

provides a second "hit" in this model, the increased tumor progression in Egfrwas mice (Figure 5B) likely occurred in response to subsequent exaggerated inflammation and dysregulated epithelial regeneration. In contrast, in the $I l 10^{-/-}$model, tumor initiation and promotion both are driven by inflammation, thus accounting for increased tumor formation and progression in $\mathrm{Il10}^{-/-}$Egfrwa5 mice. Importantly, AOM/DSS tumors were histologically distinct from those in $\mathrm{IllO}^{-/-}$mice; whereas AOM/DSS tumors were polypoid, $I l 10^{-/-}$tumors were invariably flat adenomas, which are more similar to those observed in CAC patients $(60,61)$. Moreover, the $I l 10^{-/-}$data may be more applicable to tumorigenesis in IBD patients, since these tumors develop spontaneously without mutagen administration. Thus, EGFR inactivation increases tumor progression in two distinct models of CAC, each with a unique pathological presentation.

Molecular characteristics of CAC tumors. Colorectal tumors are heterogeneous and involve the dysregulation of multiple unique and/or overlapping pathways (62). EGFR inactivation increased the pro-

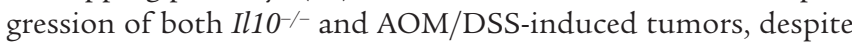
a different presentation, suggesting that tumor suppression by EGFR during inflammation is not confined to a single tumor type. To understand the molecular characteristics of these tumors, we determined the signaling pathways activated in these diverse tumors (Figure 6). All tumors in the $I l 10^{-/-}$model, once formed, showed a high level of ERK1/2 (p42/44 MAPK) phosphorylation, irrespective of EGFR status, while none showed evidence of constitutive Wnt signaling, such as nuclear $\beta$-catenin localization. In contrast, tumors from AOM/DSS-treated Egfrwas mice showed minimal ERK1/2 phosphorylation but a high level of nuclear $\beta$-catenin localization. Immunohistochemistry controls are shown in Supplemental Figure 8. These data reveal constitutive ERK1/2 activation as a feature of $\mathrm{IllO}^{-/-}$colorectal tumors, indicating that these tumors are likely driven by MAPK pathway activation. In contrast, $\mathrm{Il} \mathrm{O}^{-/}$tumors were not associated with alterations in Wnt signaling, whereas AOM/DSS tumors were likely driven by Wnt pathway activation, consistent with previous reports (63-66). This suggests that EGFR inactivation increases tumor progression across multiple tumor types during inflammation, despite differential activation of either the MAPK or Wnt pathway. There is currently debate about whether there are aberrant signaling pathways specific to CAC versus sporadic colorectal adenocarcinoma (41, 42), and further clinical studies are warranted to determine the molecular basis of these disparate tumor types. Nevertheless, we have identified ERK1/2 activation as a consistent molecular characteristic of $I l 10^{-/-}$ colorectal tumors, suggesting a potential role for dysregulation of the MAPK pathway in CAC patients with chronic inflammation, which merits further investigation.

A novel tumorigenic Braf mutation. Since $\mathrm{Il10} \mathrm{O}^{-/}$tumors showed ERK1/2 activation, irrespective of EGFR status, we determined the particular mutation driving this phenotype. We sequenced the DNA of genes involved in mouse tumor biology (Braf, Kras, and $\beta$-catenin) (63) from microdissected tumor, dysplastic, and nor-

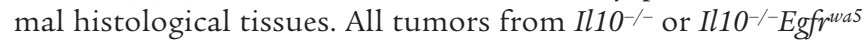
mice had a single point mutation in the Braf gene, encoding a Braf Q680H substitution (Figure 7A; equivalent to a Q609H mutation in human BRAF, human numbering used henceforth). This mutation was not observed in histologically normal colon tissue or tail DNA but was detectable in some samples from dysplastic colon tissues, suggesting that this mutation is an early event in the initiation of dysplasia and subsequent tumorigenesis. While the earliest time point at which we examined tissue samples for this mutation was 28 weeks of age, we cannot rule out that this mutation occurred at earlier ages in pre-dysplastic tissue from either the $I l 10^{-/-}$or $I l 10^{-/-}$Egfrwas mice. Nevertheless, this muta- 
A

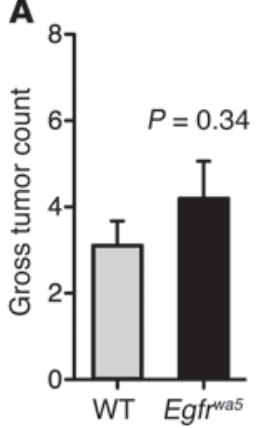

B

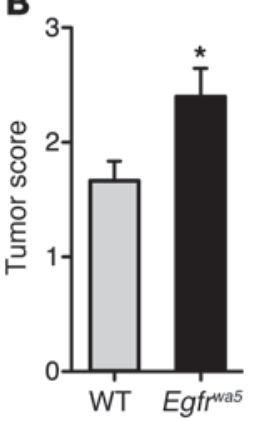

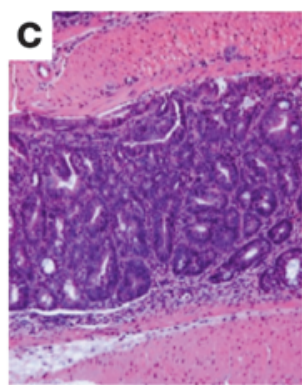

WT: low grade

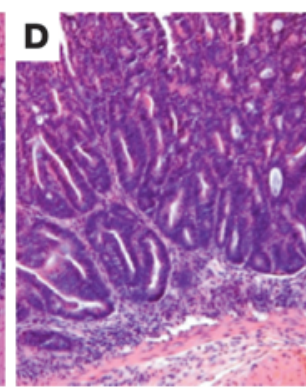

WT: high grade

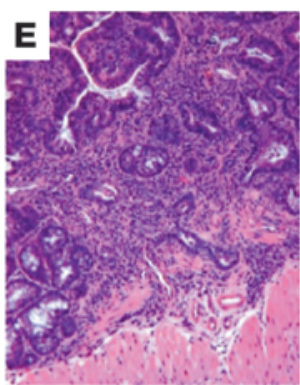

Egfrwa5: invasive

Figure 5

EGFR inhibition increases tumor progression in AOM/DSS-induced colitis. Macroscopic gross tumor counts (A) and microscopic tumor progression scores (B) from wild-type and Egfrwa5 mice in the AOM/DSS-induced CAC model; $n=5-9$; ${ }^{*}<0.05$. Scores indicate normal, 0 ; low-grade dysplasia, 1; high-grade dysplasia, 2; and invasive adenocarcinoma, 3. Representative H\&E sections showing low-grade (C) and high-grade (D) dysplasia in wild-type colons, and an invasive tumor (E) in an Egfrwa5 colon.

tion was evident in all adenomas and adenocarcinomas examined. Since Il10-1-Egfrwa5 mice developed tumors much earlier than $I l 10^{-/-}$littermates, we conclude that this mutation also occurred earlier in these mice, presumably due to increased inflammation and oxidative stress. Indeed, in addition to increased inflammation, Il10-/-Egfrwas mice also showed increased evidence of epithelial DNA damage at early ages (Figure 3D). In contrast, AOM/DSS-induced tumors from Egfrwas mice showed wild-type sequences for all genes examined, suggesting that this mutation is specific to the etiology of tumorigenesis in the $1 l 10^{-/-}$model. This $B R A F \mathrm{Q} 609 \mathrm{H}$ mutation has not been identified in human colorectal tumors, but has been reported in melanoma patients (67). Interestingly, the $\mathrm{Q} 609 \mathrm{H}$ mutation is close to the BRAF mutation commonly observed in human colorectal cancers (V600E), within a highly conserved region of the $B R A F$ activation domain (Figure 7A). Whereas the V600E mutation is an activating mutation that induces proliferation and suppresses apoptosis (68), the biochemical consequences of the Q609H mutation are unknown. To determine the functional significance of this mutation, we therefore generated mouse colon epithelial cell lines expressing vector, wild-type mouse Braf $\left(B_{\text {raf }}{ }^{W T}\right)$, or the $\mathrm{Q} 609 \mathrm{H}$ or V600E mutations (Braf ${ }^{\mathrm{Q} 609 H}$, Braf $^{\mathrm{V} 600 \mathrm{E}}$ ) and determined the influence of these Braf mutations on basal ERK1/2 phosphorylation, proliferation, and apoptosis (Figure 7, B-E). The Braf ${ }^{\mathrm{Q} 609 H}$ mutation increased basal ERK1/2 phosphorylation and proliferation and reduced basal apoptosis relative to $B r a f^{W T}$. These results were blocked with U0126, a MEK1/2 inhibitor. Furthermore, Braf ${ }^{\mathrm{Q} 609 H}$ cells were protected from apoptosis induced by TNF and IFN- $\gamma$. Therefore, this Braf ${ }^{\mathrm{Q} 609 \mathrm{H}}$ mutation confers colon epithelial cells with the tumorigenic characteristics of proliferation and survival self-sufficiency, at least partially through a MEK1/2-dependent pathway. Furthermore, Braf ${ }^{\mathrm{Q} 609 \mathrm{H}}$ was more effective than Braf ${ }^{\mathrm{V} 600 \mathrm{E}}$ in altering these characteristics; although this difference might be specific to mouse Braf, it suggests that additional factors, such as activated Rac1b (68), may be required for Braf ${ }^{V 600 E}$ to exert its full tumorigenic potential. Importantly, the V600E mutation is a major predictor of resistance to EGFR inhibition in colorectal cancer patients $(37,39)$; this Q609H mutation may represent a functional murine equivalent, given that $I l 10^{-/-}$tumors developed despite EGFR inactivation along with constitutive ERK1/2 phosphorylation. While the $\mathrm{Q} 609 \mathrm{H}$ mutation is of unclear direct significance for human colorectal cancer, these functional data are highly relevant to $B R A F^{Q 609 H}$ mutant human melanoma (67), and may represent a murine analog for studies of $B R A F^{V 600 E}$ mutant colorectal cancer. Thus, an important issue is to determine whether BRAF mutations are specifically involved in inflammation-induced colorectal tumors in patients and, subsequently, how such mutations influence EGFR inhibitor efficacy. Indeed, activating $B R A F$ mutations are known predictors for resistance to EGFR inhibitor-based chemotherapy. Moreover, BRAF inhibitors, such as vemurafenib (69), which was recently approved for treatment of metastatic melanoma, should be evaluated in clinical trials of CAC patients with the BRAFV600E mutation.

\section{Discussion}

EGFR signaling plays a central role in the regulation of colon epithelial biology and the response to injury and inflammation (Supplemental Figure 1). We have now shown for the first time to our knowledge that EGFR dramatically slows disease progression in the mouse $\mathrm{Il10}^{-/-}$spontaneous colitis model, with important implications for preventing subsequent tumorigenesis. This effect of EGFR appears to be driven by optimizing epithelial regeneration, particularly by preventing pathogenic hyperproliferation, dysplasia, and DNA damage and promoting cell survival. At the tissue level, these functions likely accelerate the rate of wound healing, thereby preventing epithelial barrier dysfunction and promoting the resolution of inflammatory injury. While the loss of EGFR signaling alone induced slight barrier dysfunction, this was not sufficient to induce colitis; however, the protective role of EGFR was readily apparent in the absence of IL-10-mediated immune tolerance. Interestingly, the increased Il10 mRNA expression in Egfrwas mice suggests a relationship between EGFR and IL-10 in the regulation of colon inflammation, which is particularly relevant given the recent appreciation of IL-10 signaling defects in subsets of IBD patients (48-51). In the absence of both EGFR and IL-10 signaling, colitis was dramatically accelerated; this was associated with an early increase in the levels of Th1/Th17 cytokine expression, without altering the specific profile of those Th1/Th17 cytokines produced. This suggests that EGFR signaling does not directly influence the type of inflammation in colitis, but rather mitigates its initiation and/or progression. While an obvious explanation is that EGFR-regulated epithelial integrity prevents the activation of 
A
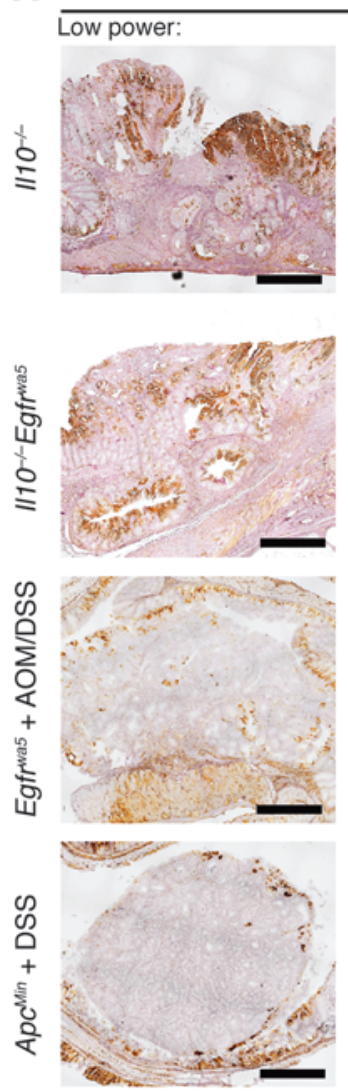

B
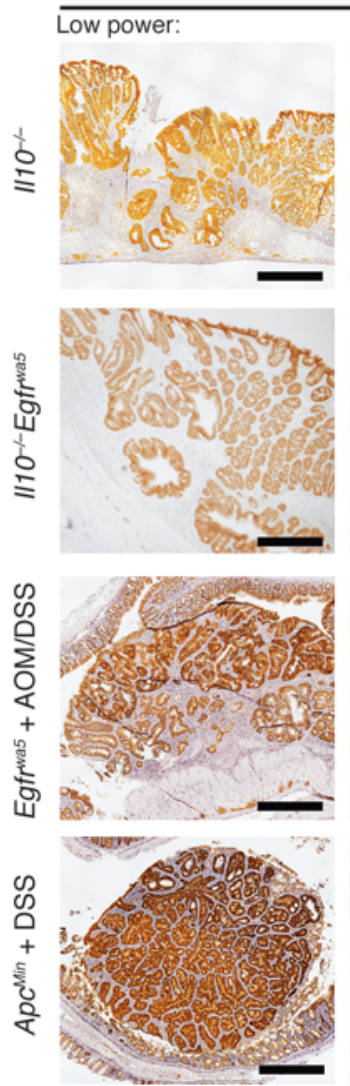

phospho-Erk1/2
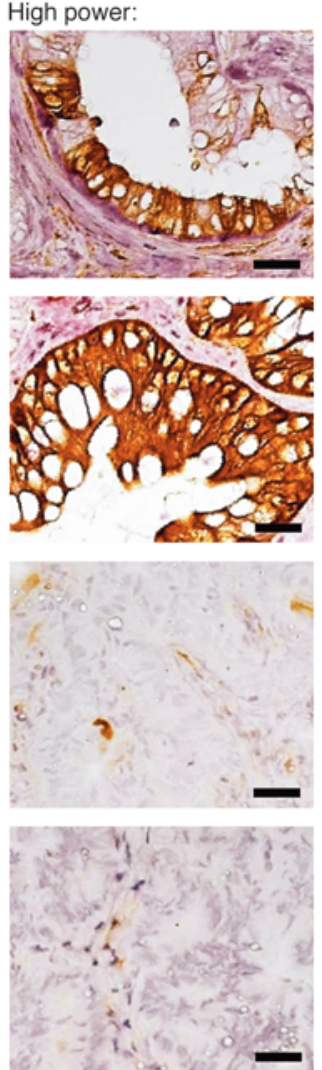

$\beta$-Catenin
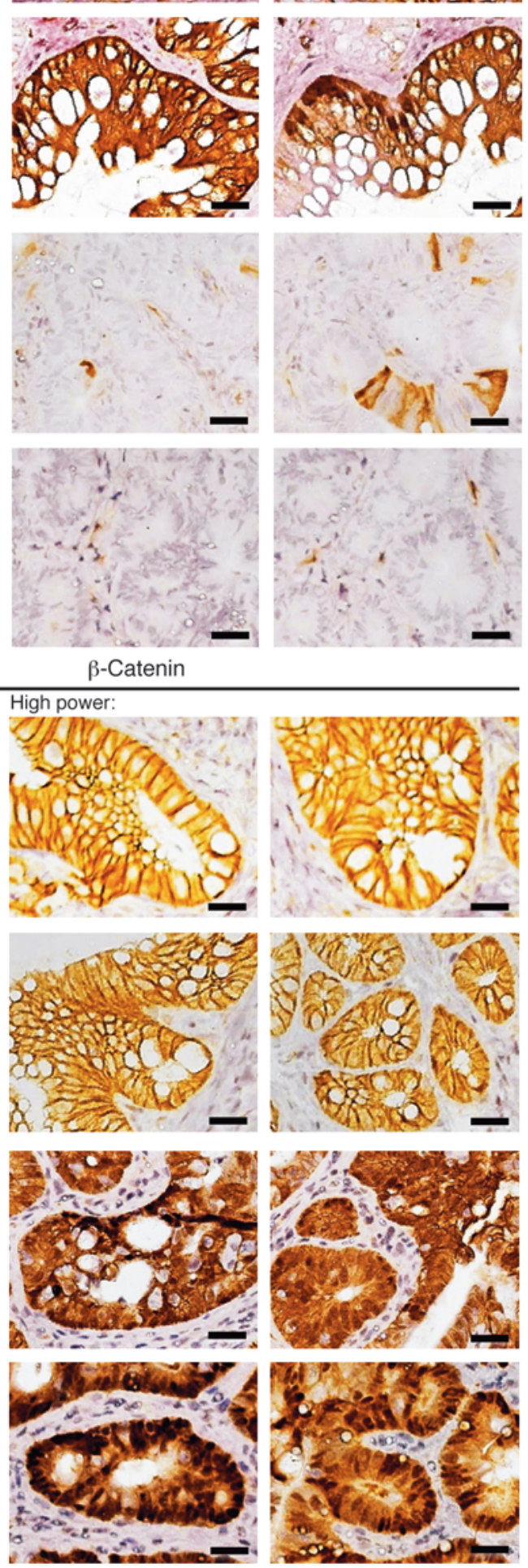

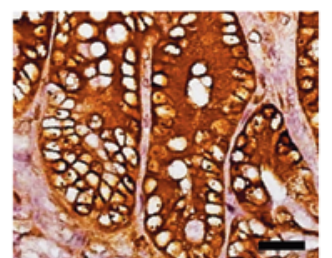

(1)
din be ineffective in this population, either due to exacerbated inflammation driving tumorigenesis or due to inherent resistance to EGFR inhibition caused by inflammation-induced mutations, potentially in the MAPK pathway. Indeed, tumors with mutant $B R A F$ or KRAS are associated with poor responses to EGFR inhibitor therapy (37-40). In the present study, the development and progression of $I l 10^{-/-}$tumors was greatly accelerated in the absence of EGFR signaling, suggesting that the identified BRAF mutation may preclude a role for EGFR in driving these tumors. Our data also suggest that EGFR signaling is important in colonic adaptive responses to injury and inflammation, involving optimization of epithelial responses and subsequent inflammation. Thus, a tumor-suppressing role for EGFR in CAC may be secondary to its ability to attenuate colitis; in particular, this may involve a reduction in IL-6/gp130 and/or IL-21 signaling, since both are linked to CAC development (70-73) and the levels of these cytokines were dramatically increased with the loss of EGFR signaling. Nevertheless, we cannot exclude a direct role for EGFR in inhibiting tumor development or growth in this model system. While a direct tumor-suppressing role for EGFR in CAC may seem counterintuitive, studies have identified similar roles for EGFR in other tumors. First, mammary and epidermoid tumor cells undergo growth arrest and apoptosis following EGF treatment $(74,75)$. Second, mutations that decrease EGFR expression in a subset of colon and gastric tumors are associated with increased tumor progression (76). Finally, activating EGFR mutations in non-small cell lung cancer are associated with 
A

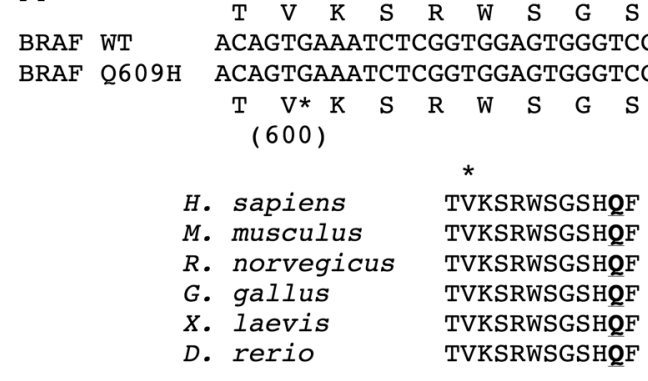

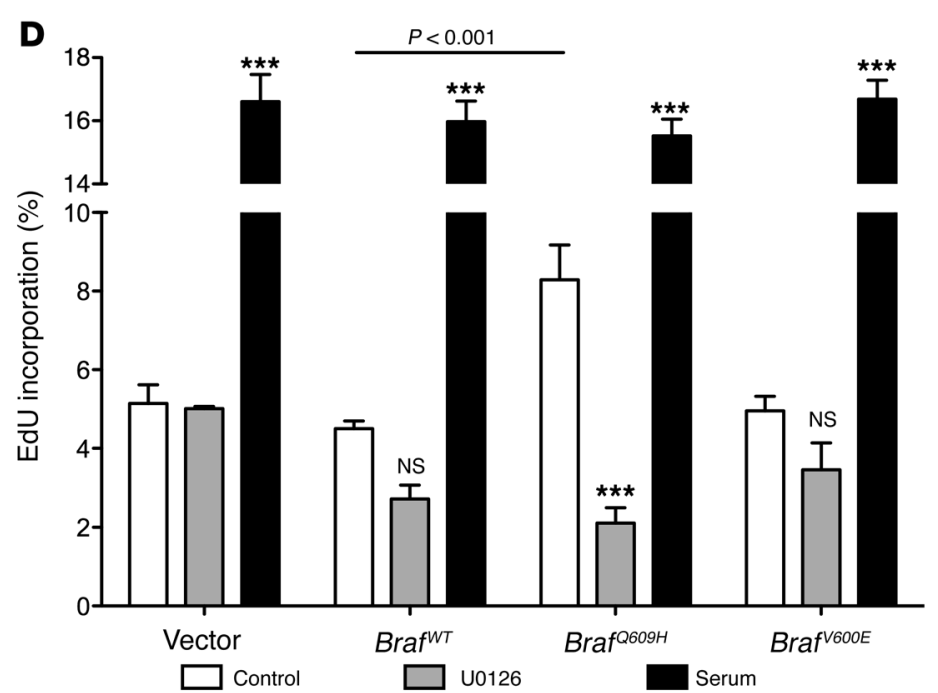

E
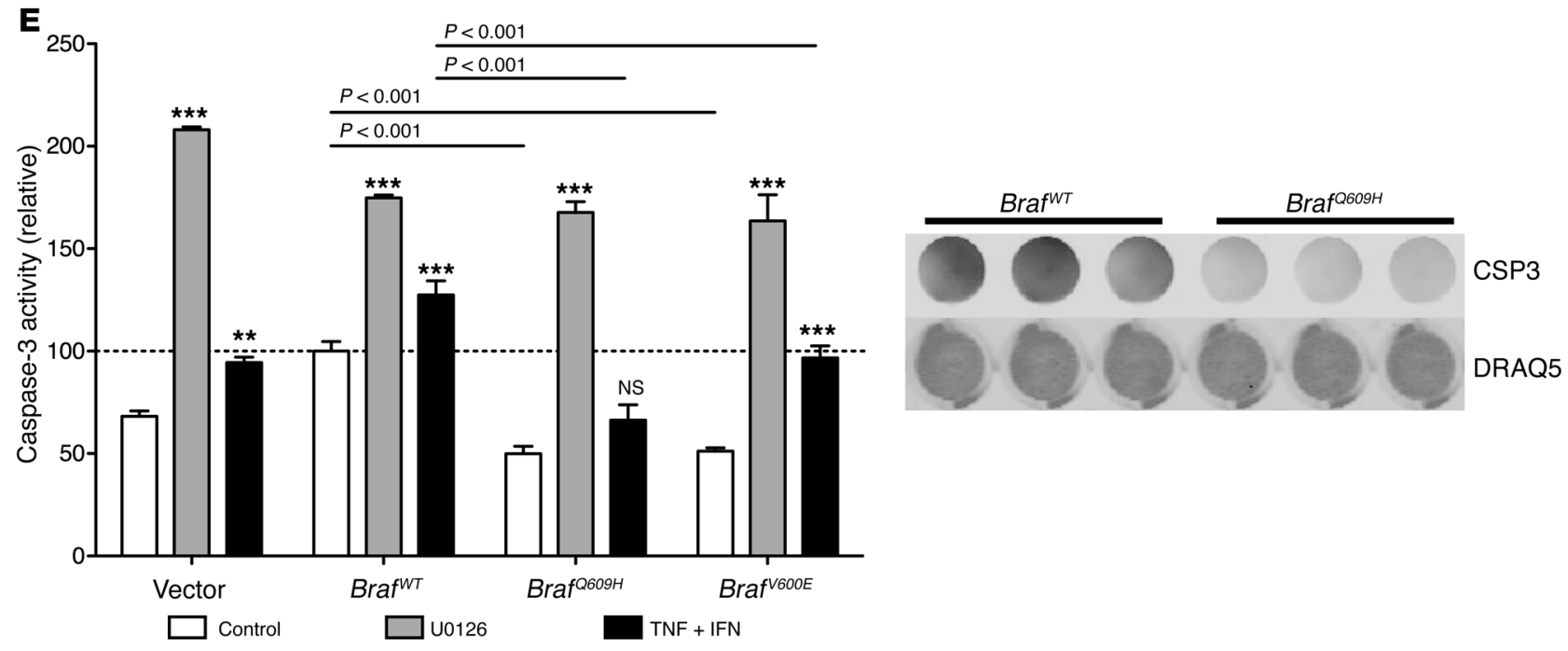


\section{Figure 7}

A novel tumorigenic Braf mutation in $/ / 10^{-/-}$colon tumors. (A) $/ / 10^{-/-}$ and $/ / 10^{-/-}$Egfrwa5 colon tumors have a single $\mathrm{G} \rightarrow \mathrm{T}$ point mutation in Braf encoding a $\mathrm{Q} 609 \mathrm{H}$ substitution in the highly conserved activation domain (human numbering noted). The V600E mutation $\left({ }^{*}\right)$ is commonly observed in human colon cancer. (B-E) YAMC cells were stably transduced with the indicated Braf mutations. (B) BRAF expression in each line, as determined by in-cell Western; cell labeling with IRDye 800 $\mathrm{NHS}$ ester was used as a loading control; $n=3$. (C) Basal levels of ERK phosphorylation, as determined by in-cell Western; total ERK was used as a loading control; $n=6$; NS, $P>0.05,{ }^{*} P<0.05$ versus $B r a f W T$. (D) $\mathrm{BrafQ609H}$ increases unstimulated cellular proliferation. Proliferative rate was determined by EdU incorporation in cells treated for 24 hours with control medium, $10 \mu \mathrm{M} \cup 0126$, or $10 \%$ fetal bovine serum. EdU incorporation was determined following a 2-hour pulse and quantified as the percentage of EdU-positive nuclei, and NuclearMask was used to stain nuclei; $n=6$; NS, $P>0.05,{ }^{* \star} P<0.001$ versus control-treated cells. Representative images of control Braf ${ }^{W T}$ and $B r a f Q 609 H$ cells are shown. Original magnification, $\times 10$. (E) Braf ${ }^{Q 609 H}$ inhibits basal and TNF + IFNinduced apoptosis. Apoptotic rate was determined by caspase-3 activity assay in cells treated for 5 hours with control medium, $10 \mu \mathrm{M}$ U0126, or $100 \mathrm{ng} / \mathrm{ml} \mathrm{TNF}+200 \mathrm{U} / \mathrm{ml} \mathrm{IFN}$. Caspase-3 activity was determined in live cells and quantified by the degradation of a specific caspase-3 substrate (CSP3); $n=3-6$; NS, $P>0.05,{ }^{* *} P<0.01$, ${ }^{\star * \star} P<0.001$ versus control-treated cells. Representative images of control BrafWT and $B r a f{ }^{Q 609 H}$ caspase-3 degradation product are shown; DRAQ5 was used a DNA dye loading control.

decreased tumor recurrence following resection (77). These observations are consistent with the findings that EGF inhibits murine colonic epithelial cell proliferation in vivo (7) and that EGF is an ineffective trophic agent for the colonic mucosa under unchallenged conditions (our unpublished observations). Interestingly, EGFR is required for Lgr5-positive intestinal epithelial stem cell growth in vitro (78). While deletion of the Apc gene in these stem cells is sufficient for intestinal tumorigenesis (79), the ability of EGFR to regulate these cells during injury, inflammation, or subsequent tumorigenesis is unknown. Although Wnt signaling is constitutively active in Lgr5-positive epithelial stem cells (80), the Ill1 $0^{-/-}$tumors in the present study did not express nuclear $\beta$-catenin; thus, we speculate that these tumors arise from a different Wnt-independent cell population, either committed epithelial progenitors or the normally quiescent Bmi1-positive intestinal epithelial stem cells that are activated upon injury $(81,82)$. Nevertheless, our finding that EGFR prevents aberrant epithelial proliferation and tumorigenesis during colitis suggests a role for EGFR in regulating epithelial stem cell or progenitor cell populations during inflammation, which could be an additional mechanism by which EGFR regulates tumor biology.

Although the major site of EGFR expression in the colon is the epithelium, several lamina propria cell types also express EGFR, including myofibroblasts and monocytic lineages (i.e., macrophages and dendritic cells) (19-21). While the functional role for EGFR in these cells is relatively unknown, each cell type is involved in both inflammation and tumor biology, suggesting that EGFR regulation of these cells may contribute to protection from colitis and CAC. In the colon, myofibroblasts regulate epithelial dynamics and inflammation and are a major stromal component of gastrointestinal tumors (83), while macrophages and dendritic cells are both important regulators and mediators of inflammation and tumor biology. Although we did not observe any striking histological differences between the tumor stroma in the present study, the potential role of EGFR in regulating these cells is an intriguing possibility. An important question is therefore how EGFR may regulate myofibroblasts, macrophage recruitment/activation, or dendritic cell infiltration with respect to inflammation and/or tumorigenesis.

In conclusion, we have shown a paradoxical role for EGFR in inhibiting inflammation and tumorigenesis in mouse models of CAC. This emphasizes the need for a molecular approach to the individualized treatment of colorectal cancer (62); in particular, the determination of BRAF and KRAS mutational status during evaluation of CAC patients, especially when considering anti-EGFR therapy. Furthermore, we showed that EGFR suppresses tumorigenesis in chronic colitis by optimizing responses to chronic inflammation and reducing subsequent tumor initiation and progression. This suggests that EGFR-targeted therapies for colitis may not be associated with increased longterm cancer risk, thereby providing an important rationale for renewed investigation of EGFR-based IBD therapies $(28,29)$. Further studies on the role of EGFR in the adaptive responses of the gastrointestinal tract to injury and inflammation will aid in the development of safer and more efficacious therapies for both IBD and CAC.

\section{Methods}

Chemicals and reagents. DSS (36-50 kDa) was from MP Biomedicals. AOM was from Wako. FITC-labeled dextran $(3-5 \mathrm{kDa})$ and inulin were from Sigma-Aldrich. Recombinant murine EGF, IFN, and TNF were from Peprotech. U0126 was from EMD Chemicals. Rat anti-Ki-67 was from Dako. Rabbit antibodies for $\mathrm{pH}-\mathrm{H} 3$, phospho-histone H2AX, phospho-ERK1/2, and $\beta$-catenin and mouse antibody for total ERK1/2 were from Cell Signaling Technology. Rabbit anti-BRAF was from Epitomics.

Animals. Wild-type (C57BL/6J), Il10-/-, Egfrwaz/wa2 , and Apc Min/+ mice were from The Jackson Laboratory; Egfrwas/+ mice were a gift from David Threadgill (University of North Carolina, Chapel Hill, North Carolina, USA). All mice were on a C57BL/ 6 background and housed under standard patho-

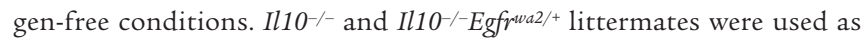
controls for $I l 10^{-/-} E g f r^{w a s}$ and $I l 10^{-/-E g f r^{w a 2} / w a 2}$ mice, respectively. Where possible, experimental and control mice were housed together. Tail DNA was genotyped using the primers listed in Supplemental Table 3. PCR for Egfrva genotyping was followed by FokI digestion (New England BioLabs).

$A O M / D S S$ tumor model. Adult mice received a single injection of AOM (12.5 mg/kg, i.p.); 5 days later, mice were given a single round of DSS ( $3 \% \mathrm{w} / \mathrm{v}$ in drinking water) for 7 days. Mice were euthanized 65 days following DSS removal.

$A p c^{\text {Min }} / D S S$ tumor model. Apc $c^{\text {Min } /+}$ mice (5 weeks old) received a single round of DSS ( $2 \% \mathrm{w} / \mathrm{v}$ in drinking water) for 1 week. Mice were euthanized at 10 weeks of age.

Tissue harvesting. After mice were euthanized, the entire cecum, colon, and rectum were removed, emptied of fecal contents, weighed, and opened longitudinally along the mesenteric border. Small longitudinal slices of mid-colon were frozen for molecular analysis. Colons were either rolled from the distal to proximal end or laid flat, and then fixed in $10 \%$ neutral-buffered formalin. Following fixation, flat colons were stained with methylene blue and examined under a dissecting microscope to quantify the gross tumors.

Histological scoring. H\&E sections of the entire cecum to rectum were scored in a manner blinded to genotype and age/treatment. For the $I l 10^{-/-}$ model, colitis scores were according to a previously published system (Supplemental Table 1) (45); tumor progression was scored based on the incidence and severity of adenomas and adenocarcinomas (Supplemental 
Table 2). Given the different morphological presentation of tumors in the AOM/DSS model, these sections were scored as normal, 0 ; low-grade dysplasia, 1 ; high-grade dysplasia, 2; or invasive adenocarcinoma, 3.

In vivo permeability measurement. To measure colon permeability, anesthetized mice were given an enema of FITC-dextran $(0.1 \mathrm{ml}$ total volume, $100 \mathrm{mg} / \mathrm{ml}$ in saline) and maintained in an inverted position until plasma samples were collected 30 minutes later. To measure entire gastrointestinal tract permeability, fasted mice were gavaged with FITC-inulin $(0.5 \mathrm{ml}$ total volume, $20 \mathrm{mg} / \mathrm{ml}$ in saline); plasma samples were collected 4 hours later. Relative plasma fluorescence was measured in a fluorometer.

Real-time RT-PCR. cDNA was synthesized from total RNA isolated from whole-thickness colon samples and analyzed by real-time RT-PCR using the primers or the primer/probe sets indicated in Supplemental Table 5. For those transcript targets using primers alone, real-time PCR was performed with iQ SYBR Green Supermix (Bio-Rad), whereas primer/probe reactions were performed with Maxima Probe qPCR master mix (Thermo Scientific). Primer/probe sets were obtained from Thermo Scientific (Solaris qPCR gene expression assays). Actin was used as an internal control for all samples, such that actin primers/SYBR green or actin primer/ probe sets were used to normalize primers/SYBR green or primer/probe transcript targets, respectively. Real-time PCR efficiency was determined by analysis of serial dilutions of colon cDNA.

Immunohistochemistry. All immunohistochemistry was performed on formalin-fixed, paraffin-embedded samples. Sections were de-waxed and rehydrated through a graded series of ethanol. Endogenous peroxidase was quenched by incubation in hydrogen peroxide (3\% w/v in methanol for 15 minutes). Antigen retrieval was performed by boiling in a pressure cooker for 10 minutes in either citrate buffer $(10 \mathrm{mM}$ citrate, $0.05 \% \mathrm{w} / \mathrm{v}$ Tween-20, $\mathrm{pH}$ 6, for Ki-67, pH-H3, phospho-histone H2AX, and $\beta$-catenin) or TrisEDTA buffer (1 mM Tris, 1 mM EDTA, 0.05\% w/v Tween-20, pH 9, for phospho-ERK1/2). Sections were blocked for 30 minutes with $10 \%$ normal goat serum (Life Technologies), prior to incubation with primary antibody for 1.5 hours at room temperature (Ki-67) or overnight at $4^{\circ} \mathrm{C}$ (all others). Rabbit antibodies were detected using HRP-labeled anti-rabbit secondary antibody (Envision, Dako), and rat anti-Ki-67 was detected using biotinylated anti-rat secondary antibody (Vector Laboratories), followed by streptavidin-labeled HRP (Life Technologies). DAB (Sigmafast, Sigma-Aldrich) was used to develop signal, and hematoxylin was used as a counterstain. Immunohistochemistry controls are shown in Supplemental Figure 8.

Epithelial proliferation, apoptosis, and DNA damage. Proliferating epithelial cells were quantified by counting Ki-67-positive epithelial cells in one-half of at least 50 well-oriented crypts from the mid- and distal colon of each animal. Metaphase epithelial cells were quantified by counting $\mathrm{pH}-\mathrm{H} 3-$ positive epithelial cells in at least 50 well-oriented crypts. Apoptotic epithelial cells were identified based on both positive TUNEL staining (ApopTag kit, Millipore) and morphological characteristics (pyknotic nuclei). Apoptotic epithelial cells were counted in an entire longitudinal cross section of the cecum, colon, and rectum and results normalized for colon length. Epithelial DNA damage was detected by staining for phospho-histone $\mathrm{H} 2 \mathrm{AX}$, an indirect marker of DNA damage. Positive epithelial cell nuclei were quantified in an entire longitudinal cross section and normalized for colon length, as in the apoptosis analyses.

Microdissection and sequencing. Tissue sections were counterstained with $\mathrm{H} \& \mathrm{E}$, and tumor and histologically normal epithelium were microdissected using a PALM Microsystem (Carl Zeiss Microscopy). Genomic DNA was isolated (QIAamp DNA Micro Kit, QIAGEN), and PCR products were generated and sequenced using the primers indicated in Supplemental Table 4. Tail DNA was used as a control to rule out germline mutations. Multiple sequence alignments were performed using CLC Sequence Viewer (CLC bio).
Generation of stable cell lines. Full-length mouse BRAF cDNA (IMAGE clone 8327547) was obtained from Open Biosystems Products. Site-directed mutagenesis (QuikChange II XL, Agilent Technologies) was used to eliminate an internal EcoRI site and then to introduce the V600E or Q609H mutation (numbers refer to human amino acid sequence); all reactions were confirmed by sequencing. Following EcoRI digestion, BRAF constructs were transferred into a retroviral vector upstream of an internal ribosomal entry site, followed by a GFP reporter (LZRS-IRES-GFP; a gift from Albert Reynolds, Vanderbilt University, Nashville, Tennessee, USA) (84); correct orientation was confirmed by BglII digestion. BRAF constructs or empty vector were transfected into ecotropic packaging cells; conditionally immortalized young adult mouse colon epithelial (YAMC) cells (85) were subsequently infected with viral supernatants, and GFP-expressing cells were sorted to produce stable cell lines transduced with equivalent levels of virus (Children's Hospital Los Angeles Flow Cytometry Core).

Cell line experiments. All experiments were conducted on YAMC cells transduced with vector or with wild-type, Q609H, or V600E Braf; experiments were performed under non-permissive conditions (cells were maintained for at least 24 hours at $37^{\circ} \mathrm{C}$ in RPMI containing $0.1 \%$ FBS prior to treatment). Control medium consisted of RPMI containing $0.1 \% \mathrm{FBS}$, $50 \mathrm{IU} / \mathrm{ml}$ penicillin, and $50 \mu \mathrm{g} / \mathrm{ml}$ streptomycin; vehicle (DMSO) was added to control medium for experiments involving U0126. BRAF expression and ERK1/2 phosphorylation were determined by In-Cell Western. Briefly, cells were fixed with paraformaldehyde ( $4 \% \mathrm{w} / \mathrm{v})$ and permeabilized with Triton X-100 $(0.1 \% \mathrm{w} / \mathrm{v})$ prior to staining with either BRAF or phospho-ERK1/2 and total ERK1/2 antibodies. Secondary antibody for BRAF and phospho-ERK1/2 was IRDye680-labeled goat anti-rabbit, and secondary antibody for total ERK1/2 was IRDye800-labeled donkey anti-mouse (LI-COR). IRDye800-NHS ester (LI-COR) was used to stain total cellular protein as a loading control for BRAF. Plates were imaged and quantified using an Odyssey Imager (LI-COR). Proliferation was determined by pulse labeling cells for 2 hours with $10 \mu \mathrm{M}$ EdU and detection using the ClickiT Alexa Fluor 555 kit (Life Technologies), according to the manufacturer's instructions. Cells were imaged on an Axio Observer microscope (Carl Zeiss Microscopy), and the percentage of EdU-positive nuclei (NuclearMask-positive cells, Life Technologies) in 2 low-power fields (average of 1,240 cells enumerated per sample) was quantified automatically using ImageJ software (http://rsb.info.nih.gov/ij/). Apoptosis was determined by an in-cell caspase-3 activity assay. Briefly, cells were incubated for 1 hour with a highly quenched caspase-3 peptide substrate (IRDye 800CW/QC-1 CSP3, LI-COR) that fluoresces following caspase-3-mediated cleavage; fluorescent product was imaged and quantified on an Odyssey Imager with the DNA dye DRAQ5 used as a loading control (Biostatus Ltd.).

Statistics. All data are presented as mean \pm SEM. Parametric methods (Student's $t$ test or ANOVA) were used for data sets that were non-categorical and homoscedastic (as confirmed by Bartlett's test). Non-parametric methods (Mann-Whitney $U$ or Kruskal-Wallis test) were used for categorical data sets (i.e., colitis scores and tumor scores), as well as with data that failed Bartlett's test for equal variance. Kaplan-Meier survival curves were compared using the log-rank test. Statistical analyses were performed using GraphPad Prism (GraphPad Software Inc.).

Study approval. Procedures were approved by the Vanderbilt University and Children's Hospital Los Angeles Institutional Animal Care and Use Committees.

\section{Acknowledgments}

The authors would like to thank David Threadgill for providing the Egfrwa5 mice. This work was supported by NIH grants R01DK056008 and R01DK54993 (to D.B. Polk), a fellowship from the Canadian Institutes of Health Research (to P.E. Dubé), 
a fellowship from the Crohn's and Colitis Foundation of America (to P.E. Dubé), and NIH award P30DK058404 to the Vanderbilt Digestive Diseases Research Center (VDDRC), including the VDDRC Histology Core laboratory.

Received for publication January 16, 2012, and accepted in revised form May 30, 2012.

1. Eaden JA, Abrams KR, Mayberry JF. The risk of colorectal cancer in ulcerative colitis: a meta-analysis. Gut. 2001;48(4):526-535.

2. Canavan C, Abrams KR, Mayberry J. Meta-analysis: colorectal and small bowel cancer risk in patients with Crohn's disease. Aliment Pharmacol Ther. 2006; 23(8):1097-1104.

3. Jess $\mathrm{T}$, et al. Risk of intestinal cancer in inflammatory bowel disease: a population-based study from olmsted county, Minnesota. Gastroenterology. 2006; 130(4):1039-1046

4. Watanabe T, Konishi T, Kishimoto J, Kotake K, Muto T, Sugihara K. Ulcerative colitis-associated colorectal cancer shows a poorer survival than sporadic colorectal cancer: a nationwide Japanese study. Inflamm Bowel Dis. 2011;17(3):802-808.

5. Laukoetter MG, et al. Intestinal cancer risk in Crohn's disease: a meta-analysis. J Gastrointest Surg. 2011;15(4):576-583.

6. Higashi D, et al. Clinical course of colorectal cancer in patients with ulcerative colitis. Anticancer Res. 2011;31(7):2499-2504.

7. Al-Nafussi AI, Wright NA. The effect of epidermal growth factor (EGF) on cell proliferation of the gastrointestinal mucosa in rodents. Virchows Arch, B, Cell Pathol. 1982;40(1):63-69.

8. Goodlad RA, Wright NA. Epidermal growth factor and transforming growth factor-alpha actions on the gut. Eur J Gastroenterol Hepatol. 1995; 7(10):928-932

9. Threadgill DW, et al. Targeted disruption of mouse EGF receptor: effect of genetic background on mutant phenotype. Science. 1995; 269(5221):230-234.

10. Helmrath MA, Erwin CR, Warner BW. A defective EGF-receptor in waved-2 mice attenuates intestinal adaptation. J Surg Res. 1997;69(1):76-80.

11. Yarden Y, Sliwkowski MX. Untangling the ErbB signalling network. Nat Rev Mol Cell Biol. 2001; 2(2):127-137.

12. Egger B, Büchler MW, Lakshmanan J, Moore P, Eysselein VE. Mice harboring a defective epidermal growth factor receptor (waved-2) have an increased susceptibility to acute dextran sulfate-induced colitis. Scand J Gastroenterol. 2000;35(11):1181-1187.

13. Duh G, Mouri N, Warburton D, Thomas DW. EGF regulates early embryonic mouse gut development in chemically defined organ culture. Pediatr Res. 2000; 48(6):794-802.

14. Frey MR, Golovin A, Polk DB. Epidermal growth factor-stimulated intestinal epithelial cell migration requires Src family kinase-dependent p38 MAPK signaling. J Biol Chem. 2004;279(43):44513-44521.

15. Yamaoka T, et al. Transactivation of EGF receptor and ErbB2 protects intestinal epithelial cells from TNF-induced apoptosis. Proc Natl Acad Sci U S A. 2008;105(33):11772-11777.

16. McCole DF, Barrett KE. Decoding epithelial signals: critical role for the epidermal growth factor receptor in controlling intestinal transport function. Acta Physiol (Oxf). 2009;195(1):149-159.

17. Brandl K, et al. MyD88 signaling in nonhematopoietic cells protects mice against induced colitis by regulating specific EGF receptor ligands. Proc Natl Acad Sci U S A. 2010;107(46):19967-19972.

18. Yan F, et al. Colon-specific delivery of a probiotic-derived soluble protein ameliorates intestinal inflammation in mice through an EGFR-dependent

Address correspondence to: D. Brent Polk, Children's Hospital Los Angeles, 4650 Sunset Boulevard MS\#126, Los Angeles, California 90027, USA. Phone: 323.361.2278; Fax: 323.361.3719; E-mail: dbpolk@chla.usc.edu.

Steven J. McElroy's present address is: Department of Pediatrics, University of Iowa, Iowa City, Iowa, USA.

mechanism. J Clin Invest. 2011;121(6):2242-2253.

19. Playford RJ, Hanby AM, Gschmeissner S, Peiffer LP, Wright NA, McGarrity T. The epidermal growth factor receptor (EGF-R) is present on the basolateral, but not the apical, surface of enterocytes in the human gastrointestinal tract. Gut. 1996; 39(2):262-266.

20. Yoo J, Perez CE, Nie W, Edwards RA, Sinnett-Smith J, Rozengurt E. TNF-alpha induces upregulation of EGFR expression and signaling in human colonic myofibroblasts. Am J Physiol Gastrointest Liver Physiol. 2012;302(8):G805-G814.

21. Eales-Reynolds LJ, Laver H, Modjtahedi H, Mojtahedi $\mathrm{H}$. Evidence for the expression of the EGF receptor on human monocytic cells. Cytokine. 2001; 16(5):169-172.

22. Cohen S, Carpenter G. Human epidermal growth factor: isolation and chemical and biological properties. Proc Natl Acad Sci U S A. 1975;72(4):1317-1321.

23. Heitz PU, Kasper M, van Noorden S, Polak JM, Gregory H, Pearse AG. Immunohistochemical localisation of urogastrone to human duodenal and submandibular glands. Gut. 1978;19(5):408-413.

24. Wright NA, Pike C, Elia G. Induction of a novel epidermal growth factor-secreting cell lineage by mucosal ulceration in human gastrointestinal stem cells. Nature. 1990;343(6253):82-85.

25. Fukata $M$, et al. Toll-like receptor- 4 promotes the development of colitis-associated colorectal tumors. Gastroenterology. 2007;133(6):1869-1881.

26. Alexander RJ, Panja A, Kaplan-Liss E, Mayer L, Raicht RF. Expression of growth factor receptor-encoded mRNA by colonic epithelial cells is altered in inflammatory bowel disease. Dig Dis Sci. 1995;40(3):485-494.

27. Babyatsky MW, Rossiter G, Podolsky DK. Expression of transforming growth factors alpha and beta in colonic mucosa in inflammatory bowel disease. Gastroenterology. 1996;110(4):975-984.

28. Sinha A, Nightingale J, West KP, Berlanga-Acosta J, Playford RJ. Epidermal growth factor enemas with oral mesalamine for mild-to-moderate left-sided ulcerative colitis or proctitis. N Engl J Med. 2003; 349(4):350-357.

29. Farrell RJ. Epidermal growth factor for ulcerative colitis. N Engl J Med. 2003;349(4):395-397.

30. Cohen G, et al. Epidermal growth factor receptor signaling is up-regulated in human colonic aberrant crypt foci. Cancer Res. 2006;66(11):5656-5664.

31. Goldstein NS, Armin M. Epidermal growth factor receptor immunohistochemical reactivity in patients with American Joint Committee on Cancer Stage IV colon adenocarcinoma: implications for a standardized scoring system. Cancer. 2001; 92(5):1331-1346.

32. Svrcek M, Cosnes J, Tiret E, Bennis M, Parc Y, Fléjou JF. Expression of epidermal growth factor receptor (EGFR) is frequent in inflammatory bowel disease (IBD)-associated intestinal cancer. Virchows Arch. 2007;450(2):243-244.

33. Roberts RB, et al. Importance of epidermal growth factor receptor signaling in establishment of adenomas and maintenance of carcinomas during intestinal tumorigenesis. Proc Natl Acad Sci U S A. 2002; 99(3):1521-1526.

34. Dougherty U, et al. Epidermal growth factor receptor is required for colonic tumor promotion by dietary fat in the azoxymethane/dextran sulfate sodium model: roles of transforming growth factor-\{alpha\} and PTGS2. Clin Cancer Res. 2009; 15(22):6780-6789.

35. Alferez D, et al. Dual inhibition of VEGFR and EGFR signaling reduces the incidence and size of intestinal adenomas in $\mathrm{Apc}(\mathrm{Min} /+)$ mice. Mol Cancer Ther. 2008;7(3):590-598

36. Fichera A, et al. Epidermal growth factor receptor signaling is required for microadenoma formation in the mouse azoxymethane model of colonic carcinogenesis. Cancer Res. 2007;67(2):827-835.

37. Wheeler DL, Dunn EF, Harari PM. Understanding resistance to EGFR inhibitors-impact on future treatment strategies. Nat Rev Clin Oncol. 2010; 7(9):493-507.

38. Lievre A, et al. KRAS mutation status is predictive of response to cetuximab therapy in colorectal cancer. Cancer Res. 2006;66(8):3992-3995.

39. Laurent-Puig P, et al. Analysis of PTEN, BRAF, and EGFR status in determining benefit from cetuximab therapy in wild-type KRAS metastatic colon cancer. J Clin Oncol. 2009;27(35):5924-5930.

40. Bardelli A, Siena S. Molecular mechanisms of resistance to cetuximab and panitumumab in colorectal cancer. J Clin Oncol. 2010;28(7):1254-1261.

41. Aust DE, et al. Mutations of the BRAF gene in ulcerative colitis-related colorectal carcinoma. Int J Cancer. 2005;115(5):673-677.

42. Sanchez JA, Dejulius KL, Bronner M, Church JM, Kalady MF. Relative role of methylator and tumor suppressor pathways in ulcerative colitis-associated colon cancer. Inflamm Bowel Dis. 2011; 17(9):1966-1970.

43. Kühn R, Löhler J, Rennick D, Rajewsky K, Müller W. Interleukin-10-deficient mice develop chronic enterocolitis. Cell. 1993;75(2):263-274.

44. Berg DJ, et al. Enterocolitis and colon cancer in interleukin-10-deficient mice are associated with aberrant cytokine production and CD4(+) TH1like responses. J Clin Invest. 1996;98(4):1010-1020.

45. Kennedy RJ, Hoper M, Deodhar K, Erwin PJ, Kirk SJ, Gardiner KR. Interleukin 10-deficient colitis: new similarities to human inflammatory bowel disease. Br J Surg. 2000;87(10):1346-1351.

46. Neufert C, Becker C, Neurath MF. An inducible mouse model of colon carcinogenesis for the anal$y$ sis of sporadic and inflammation-driven tumor progression. Nature Protocols. 2007;2(8):1998-2004.

47. Yan F, John SK, Wilson G, Jones DS, Washington MK, Polk DB. Kinase suppressor of Ras-1 protects intestinal epithelium from cytokine-mediated apoptosis during inflammation. J Clin Invest. 2004; 114(9):1272-1280.

48. Franke A, et al. Sequence variants in IL10, ARPC2 and multiple other loci contribute to ulcerative colitis susceptibility. Nat Genet. 2008;40(11):1319-1323.

49. Glocker EO, et al. Inflammatory bowel disease and mutations affecting the interleukin-10 receptor. NEngl J Med. 2009;361(21):2033-2045.

50. Amre DK, et al. Interleukin 10 (IL-10) gene variants and susceptibility for paediatric onset Crohn's disease. Aliment Pharmacol Ther. 2009;29(9):1025-1031.

51. Glocker EO, et al. Infant colitis - it's in the genes. Lancet. 2010;376(9748):1272.

52. Lee $\mathrm{D}$, et al. Wa5 is a novel ENU-induced antimorphic allele of the epidermal growth factor receptor. Mamm Genome. 2004;15(7):525-536.

53. Sellon RK, et al. Resident enteric bacteria are necessary for development of spontaneous colitis and 
immune system activation in interleukin-10-deficient mice. Infect Immun. 1998;66(11):5224-5231.

54. Su L, et al. Targeted epithelial tight junction dysfunction causes immune activation and contributes to development of experimental colitis. Gastroenterology. 2009;136(2):551-563.

55. Luetteke NC, et al. The mouse waved- 2 phenotype results from a point mutation in the EGF receptor tyrosine kinase. Genes Dev. 1994;8(4):399-413.

56. Kaiser GC, Polk DB. Tumor necrosis factor alpha regulates proliferation in a mouse intestinal cell line. Gastroenterology. 1997;112(4):1231-1240.

57. Rogakou EP, Pilch DR, Orr AH, Ivanova VS, Bonner WM. DNA double-stranded breaks induce histone H2AX phosphorylation on serine 139. J Biol Chem. 1998;273(10):5858-5868.

58. Papanikolaou A, Wang QS, Delker DA, Rosenberg DW. Azoxymethane-induced colon tumors and aberrant crypt foci in mice of different genetic susceptibility. Cancer Lett. 1998;130(1-2):29-34.

59. Khan N, Afaq F, Mukhtar H. Lifestyle as risk factor for cancer: Evidence from human studies. Cancer Lett. 2010;293(2):133-143.

60. Ullman TA, Loftus EV Jr, Kakar S, Burgart LJ, Sandborn WJ, Tremaine WJ. The fate of low grade dysplasia in ulcerative colitis. Am J Gastroenterol. 2002; 97(4):922-927.

61. Ullman T, Croog V, Harpaz N, Sachar D, Itzkowitz S Progression of flat low-grade dysplasia to advanced neoplasia in patients with ulcerative colitis. Gastroenterology. 2003;125(5):1311-1319.

62. Pritchard CC, Grady WM. Colorectal cancer molecular biology moves into clinical practice. Gut. 2011; 60(1):116-129.

63. Uronis JM, Herfarth HH, Rubinas TC, Bissahoyo AC, Hanlon K, Threadgill DW. Flat colorectal cancers are genetically determined and progress to invasion without going through a polypoid stage. Cancer Res. 2007;67(24):11594-11600.
64. Uronis JM, Threadgill DW. Murine models of colorectal cancer. Mamm Genome. 2009;20(5):261-268.

65. Tanaka T, Kohno H, Suzuki R, Yamada Y, Sugie $\mathrm{S}$, Mori H. A novel inflammation-related mouse colon carcinogenesis model induced by azoxymethane and dextran sodium sulfate. Cancer Sci. 2003; 94(11):965-973.

66. Gerling M, et al. Characterization of chromosomal instability in murine colitis-associated colorectal cancer. PLoS One. 2011;6(7):e22114.

67. Casula M, et al. BRAF gene is somatically mutated but does not make a major contribution to malignant melanoma susceptibility: the Italian Melanoma Intergroup Study. J Clin Oncol. 2004; 22(2):286-292

68. Matos P, et al. B-Raf(V600E) cooperates with alternative spliced Rac1b to sustain colorectal cancer cell survival. Gastroenterology. 2008;135(3):899-906.

69. Flaherty KT, Yasothan U, Kirkpatrick P. Vemurafenib. Nature Reviews Drug Discovery. 2011; 10(11):811-812.

70. Becker C, et al. TGF-beta suppresses tumor progression in colon cancer by inhibition of IL- 6 trans-signaling. Immunity. 2004;21(4):491-501.

71. Grivennikov S, et al. IL-6 and Stat 3 are required for survival of intestinal epithelial cells and development of colitis-associated cancer. Cancer Cell. 2009; 15(2):103-113.

72. Stolfi C, et al. Involvement of interleukin- 21 in the regulation of colitis-associated colon cancer. J Exp Med. 2011;208(11):2279-2290.

73. Jauch D, et al. Interleukin 21 controls tumour growth and tumour immunosurveillance in colitis-associated tumorigenesis in mice. Gut. 2011; 60(12):1678-1686.

74. Tikhomirov O, Carpenter G. Ligand-induced, p38-dependent apoptosis in cells expressing high levels of epidermal growth factor receptor and ErbB-2. J Biol Chem. 2004;279(13):12988-12996.
75. Song JY, Lee SW, Hong JP, Chang SE, Choe H, Choi J. Epidermal growth factor competes with EGF receptor inhibitors to induce cell death in EGFR-overexpressing tumor cells. Cancer Lett. 2009; 283(2):135-142.

76. Baranovskaya S, et al. Down-regulation of epidermal growth factor receptor by selective expansion of a $5^{\prime}$-end regulatory dinucleotide repeat in colon cancer with microsatellite instability. Clinical Cancer Research. 2009;15(14):4531-4537.

77. Lee YJ, et al. Activating mutations within the EGFR kinase domain: a molecular predictor of disease-free survival in resected pulmonary adenocarcinoma. J Cancer Res Clin Oncol. 2009;135(12):1647-1654.

78. Sato T, et al. Single Lgr5 stem cells build crypt-villus structures in vitro without a mesenchymal niche. Nature. 2009;459(7244):262-265.

79. Barker N, et al. Crypt stem cells as the cells-of-origin of intestinal cancer. Nature. 2009;457(7229):608-611.

80. Barker $\mathrm{N}$, et al. Identification of stem cells in small intestine and colon by marker gene Lgr5. Nature. 2007; 449(7165):1003-1007.

81. Sangiorgi E, Capecchi MR. Bmi1 is expressed in vivo in intestinal stem cells. Nat Genet. 2008; 40(7):915-920.

82. Yan KS, et al. The intestinal stem cell markers Bmi1 and Lgr5 identify two functionally distinct populations. Proc Natl Acad Sci U S A. 2012;109(2):466-471.

83. Powell DW, Mifflin RC, Valentich JD, Crowe SE, Saada JI, West AB. Myofibroblasts. II. Intestinal subepithelial myofibroblasts. Am J Physiol. 1999; 277(2 pt 1):C183-C201.

84. Ireton RC, et al. A novel role for p120 catenin in E-cadherin function. J Cell Biol. 2002;159(3):465-476.

85. Whitehead RH, VanEeden PE, Noble MD, Ataliotis P, Jat PS. Establishment of conditionally immortalized epithelial cell lines from both colon and small intestine of adult $\mathrm{H}-2 \mathrm{~Kb}-\mathrm{tsA} 58$ transgenic mice. Proc Natl Acad Sci U S A. 1993;90(2):587-591. 